\title{
Structure and physical properties of paracrystalline atomistic models of amorphous silicon
}

\author{
P. M. Voyles ${ }^{\text {a) }}$ \\ Department of Physics, University of Illinois, 1110 W. Green Street, Urbana, Illinois 61801 \\ and NEC Research Institute, 4 Independence Way, Princeton, New Jersey 08540 \\ N. Zotov ${ }^{\text {b) }}$ \\ Bayerisches Geoinstitut, Universitat Bayreuth, D-95440 Bayreuth, Germany \\ S. M. Nakhmanson ${ }^{\text {c) }}$ and D. A. Drabold \\ Department of Physics and Astronomy, Condensed Matter and Surface Science Program, Ohio University, \\ Athens, Ohio 45701-2979 \\ J. M. Gibson \\ Materials Science Division, Argonne National Laboratory, 9700 Cass Avenue, Argonne, Illinois 60439 \\ M. M. J. Treacy \\ NEC Research Institute, 4 Independence Way, Princeton, New Jersey 08540 \\ P. Keblinski \\ Department of Materials Science and Engineering, Rensselaer Polytechnic Institute, 110 8th Street, \\ MRC 115, Troy, New York 12180-3590
}

(Received 31 May 2001; accepted for publication 1 August 2001)

\begin{abstract}
We have examined the structure and physical properties of paracrystalline molecular dynamics models of amorphous silicon. Simulations from these models show qualitative agreement with the results of recent mesoscale fluctuation electron microscopy experiments on amorphous silicon and germanium. Such agreement is not found in simulations from continuous random network models. The paracrystalline models consist of topologically crystalline grains which are strongly strained and a disordered matrix between them. We present extensive structural and topological characterization of the medium range order present in the paracrystalline models and examine their physical properties, such as the vibrational density of states, Raman spectra, and electron density of states. We show by direct simulation that the ratio of the transverse acoustic mode to transverse optical mode intensities $I_{\mathrm{TA}} / I_{\mathrm{TO}}$ in the vibrational density of states and the Raman spectrum can provide a measure of medium range order. In general, we conclude that the current paracrystalline models are a good qualitative representation of the paracrystalline structures observed in the experiment and thus provide guidelines toward understanding structure and properties of medium-range-ordered structures of amorphous semiconductors as well as other amorphous materials. (C) 2001 American Institute of Physics. [DOI: 10.1063/1.1407319]
\end{abstract}

\section{INTRODUCTION}

The impetus for the paracrystalline model of amorphous semiconductors arose from our attempts to determine the degree of medium range order present in these materials. Diffraction measurements ${ }^{1-3}$ show that $a-\mathrm{Si}, a-\mathrm{Ge}$, and other materials that form tetrahedral covalently bonded crystals maintain the same pattern of four nearest neighbors in the amorphous phase, but do not have the long-range translational order that leads to sharp diffraction maxima.

There have been tantalizing glimpses of medium range order (MRO) at length scales on the order of $10 \AA$, between

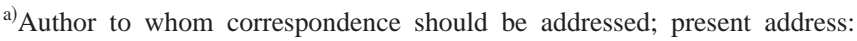
Bell Laboratories, 700 Mountain Ave., Murray Hill, NJ 07079; electronic mail: pvoyles@bell-labs.com

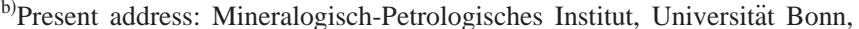
Poppelsdorfer Schloss, D-53115 Bonn, Germany.

${ }^{c}$ Present address: Dept. of Physics, North Carolina State University, Raleigh, NC 27695-8202.
}

the short range order and long range disorder fixed by diffraction. These glimpses come from the first sharp diffraction peak, ${ }^{4}$ more local probes of the material like nuclear magnetic resonance ${ }^{5}$ and near-edge $\mathrm{x}$-ray absorption fine structure, ${ }^{6}$ and especially probes of the vibrational spectrum such as neutron scattering ${ }^{7,8}$ and Raman spectroscopy. ${ }^{9,10}$ Unfortunately, interpreting these measurements in terms of a specific sample structure has proven elusive.

One of the most successful avenues of approach to the structure of amorphous semiconductors has been the creation of computer generated models. The end-products of many of these modeling efforts have been continuous random networks (CRNs), a model of amorphous structure first proposed over 50 years ago by Zachariasen. ${ }^{11} \mathrm{CRN}$ computer models have successfully reproduced the diffraction signature and the electron and vibrational densities of states of amorphous silicon. $^{12}$

The CRN model does not, however, capture the degree of MRO that has been observed ${ }^{13}$ in as-deposited amorphous 
semiconductor thin films using fluctuation electron microscopy. ${ }^{14}$ Fluctuation microscopy provides information which depends on the four-body pair-pair correlation function $^{15} g_{4}\left(r_{1}, r_{2}, r, \theta\right)$, which has been shown to be much more sensitive to MRO than the pair correlation function $g_{2}(r)$ yielded by diffraction. ${ }^{16}$ Fluctuation microscopy on thin films of $a$-Si and $a$-Ge indicate that both materials have significant ordering on a length scale of $\sim 15 \AA,{ }^{14}$ and that this order decays on thermal annealing ${ }^{17}$ (for $a-\mathrm{Ge}$ ) and on exposure to light ${ }^{18}$ (for hydrogenated amorphous silicon).

In contrast to the CRN model, our recently proposed paracrystalline (PC) model of amorphous semiconductors exhibits MRO that is in qualitative agreement with the fluctuation microscopy data. ${ }^{13}$ A PC structure consists of small grains, less than $30 \AA$ in diameter, which have topologically crystalline bonding but within which the atoms are significantly displaced from their crystalline lattice positions by strain from the grain boundaries. ${ }^{13,19}$ The grains are separated by a more disordered matrix. Our PC model is similar to the earlier microcrystalline model. ${ }^{20}$ However, this earlier model failed to reproduce the diffraction spectrum, ${ }^{21}$ which can be attributed to the lack of elastic deformation of the small crystalline grains. The atomistic computational techniques used to generate the PC model structures include the effects of strain, and thus provide structures that can appear amorphous to diffraction but also be topologically crystalline, provided the grains are small enough $(<30 \AA)$.

In this article we extensively analyze the structure and various physical properties of five computer-generated PC models with varying PC grain size. In Sec. II we briefly summarize the molecular dynamics (MD) based method used to generate our models. In Sec. III, we present the structural and topological analysis of our models. In particular, we identify the topologically crystalline PC grains using Schläfli cluster analysis ${ }^{19}$ and show that the PC grains are distinctly more ordered than the surrounding matrix. Section IV is devoted to vibrational properties and includes calculations of the vibrational density of states (VDOS), the Raman spectra, and the partial Raman spectra of the PC grains. In particular, since our models have controlled and well-characterized variations in MRO, we demonstrate by direct simulation that the ratio of the transverse acoustic to transverse optic intensities $I_{\mathrm{TA}} / I_{\mathrm{TO}}$ of the vibrational density of states and the Raman spectra provides a measure of MRO. In Sec. $V$ we present the electron density of states (EDOS) of the models. Finally, Sec. VI reports the conclusions from our analysis of the PC models.

\section{MOLECULAR DYNAMICS MODELING}

Our approach to the synthesis of model PC material is based on the method of synthesis from the melt developed by Keblinski and co-workers in the context of simulation of nanocrystalline material and uses their specialized MD program. $^{22}$ The first three models were synthesized using the Stillinger and Weber ${ }^{23}$ (SW) empirical Si potential. The welltested SW interatomic potential provides not only a good description of the crystalline and amorphous phases, ${ }^{24}$ but also of the six-coordinated structure of the liquid. The melt- ing temperature of $1690 \pm 20 \mathrm{~K}$ observed in simulations is also a good match for the experimental value of $1683 \mathrm{~K}$. Unfortunately, the SW potential models exhibit a high density of coordination defects, as discussed in Sec. III A. This is also observed in CRN models synthesized by quenching from the melt with the SW potential. In CRNs, this difficulty is somewhat mitigated by using the Bazant-Kaxiras environment-dependent interatomic potential (EDIP) ${ }^{25}$ during synthesis instead of SW, so we have also synthesized two models using EDIP. These models have far fewer coordination defects. Using two potentials also allows a rough test of which of the results are potential dependent.

As in the previous simulations, ${ }^{22}$ we started by arranging four spherical crystalline seeds on the fcc sites of the a threedimensional (3D) periodic cubic simulation supercell by designating atoms in the grains from a perfect $\mathrm{Si}$ crystal. The synthesis recipe with the SW potential is as follows: First, the crystal is melted by heating it well above the melting temperature to $T=3000 \mathrm{~K}$ at constant volume for $1000 \mathrm{MD}$ steps while the seed atoms are kept fixed at their perfectcrystal positions. We used a MD time step of $2 \times 10^{-15} \mathrm{~s}$ throughout. In the next $1000 \mathrm{MD}$ steps the four seeds are gradually rotated through random angles about random directions. After further equilibration at high temperature, the system is cooled down to $T=1100 \mathrm{~K}$ and a constant-pressure algorithm is applied to relax the system to zero external pressure. From then on the system is allowed to evolve freely with no constraints imposed on the seed atoms. The synthesis continues until the internal energy and density of the system stop decreasing. This synthesis phase typically requires 300000 time steps. These rather lengthy simulations are needed to relax the dense and overcoordinated liquid structure into a less dense amorphous structure. Next the system is cooled down under zero external pressure by 200000 time step runs at $T=1000,950$, and $900 \mathrm{~K}$ allowing further relaxation. Below $900 \mathrm{~K}$ the mobility of $\mathrm{Si}$ atoms becomes essentially zero (at least at the MD time scale), so a final relaxation at $T=0 \mathrm{~K}$ for $\sim 20000 \mathrm{MD}$ steps completes the synthesis process. The synthesis recipe with EDIP is similar, but the temperatures are different because the melting temperature for EDIP is $\sim 160 \mathrm{~K}$ lower than for the SW potential. $^{26}$ Therefore, following the high-temperature melting and seed rotation steps, we quenched the system to $T$ $=900 \mathrm{~K}$ and relaxed further at lower temperatures.

All the models start with four seed crystals. The EDIP models contain 512 atoms, the SW models 512, 700, and 1000 atoms. In the 512 atom structures, the seed centers are separated by $2.82 a_{0}$, where $a_{0}$ is the diamond crystal lattice parameter of $\mathrm{Si}, 5.430 \AA$. The seeds in the 700 and 1000 atom structures are separated by $3.13 a_{0}$ and $3.54 a_{0}$, respectively. When used to grow polycrystalline silicon with larger crystalline grains $(30-100 \AA)$, this method generated structures with signatures of crystallinity in their pair correlation function that would be detected by diffraction. When the grain size is smaller, as in the structures described here, the material looks less like a crystal in diffraction (see Sec. III A). A CRN structure was also created for comparison using the SW potential and a "slow" (i.e., involving several 
million MD steps) quench from the melt, but without the presence of any seed crystals.

We have also generated larger models using the SW potential with the same average seed spacings but containing 24 randomly rotated crystalline seeds and eight times as many atoms. Each of these models has a statistically similar structure to the corresponding smaller model and exhibited similar medium-range order characteristics. Since we wish to perform vibrational and electronic spectra characterizations which are computationally demanding, in the rest of this article we focus on thorough characterization of the four-grain models.

As in our previous work, ${ }^{13,19}$ each model is named with a type (PC or CRN), the creator's initial (in this case all $\mathrm{K}$ for Keblinski) and an identifying number. In order of increasing system and seed crystal size, we name the SW PC models PC-K1, PC-K1.5, and PC-K2. PC-K1 and PC-K2 have been examined previously under the same names. ${ }^{13,19}$ The CRN structure is called CRN-K1. The two 512 atom structures synthesized with EDIP are called PC-K1EDIP1 and PC-K1EDIP2. PC-K1.5 and the EDIP structures have not been previously considered. All of the SW potential models and PC-K1EDIP1 start with $25 \%$ of the atoms in the crystal seeds; PC-K1EDIP2 starts with $50 \%$.

As we will demonstrate, these models of PC material capture basic structural features exhibited by experimental samples. However, as already mentioned, structures prepared with the SW potential are overcoordinated, which leads to unphysical effects due to the excessively high density of defects. Therefore, before calculating the electronic properties of the SW models, we relaxed and annealed them with EDIP in an attempt to reduce the density of coordination defects to more realistic levels. This treatment did not alter in any significant manner the PC character of the models as analyzed in Sec. III. Nor did a relaxation with the Kirkwood potential $^{27}$ (which did not allow bond switching) performed prior to calculating the vibrational properties. This indicates that the PC structure is robust in several potentials.

\section{SPATIAL STRUCTURE AND TOPOLOGY}

In this section we examine the structure of the models. First we demonstrate that the short-range order of the models is realistic. Then we characterize the MRO of the models by fluctuation microscopy simulations and by examining their topology to identify the topologically crystalline PC grains. Finally, we examine the structure of just the PC grains. We

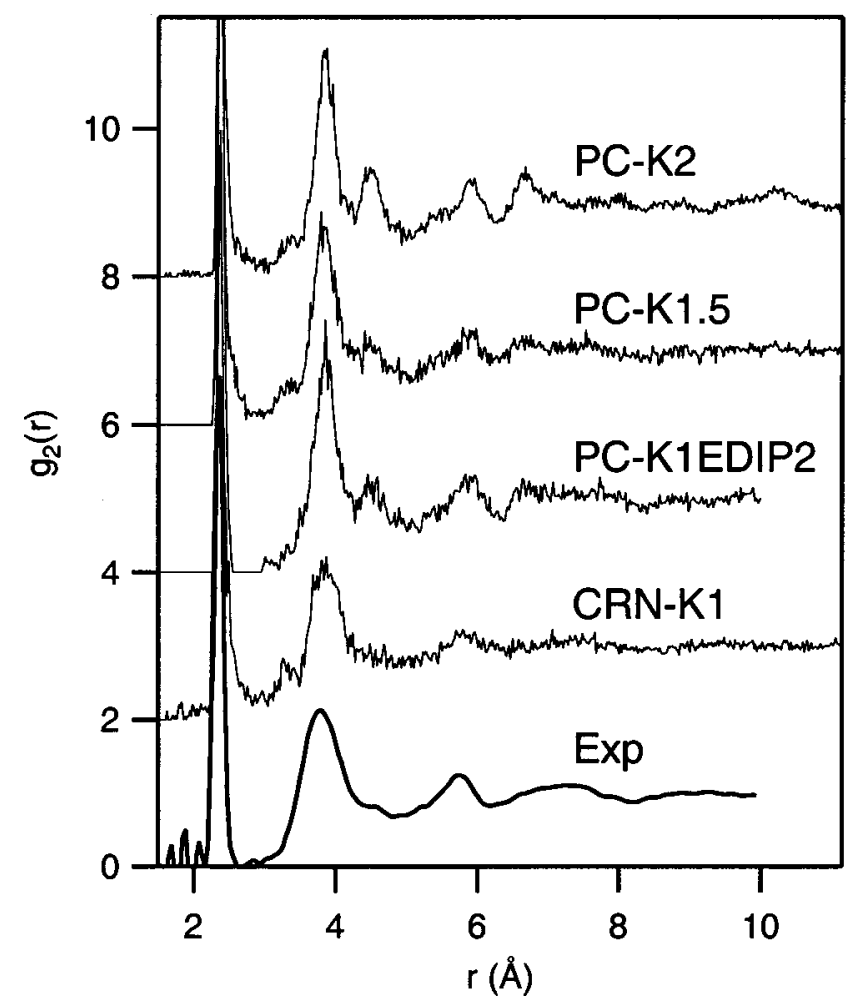

FIG. 1. $g_{2}(r)$ of three PC models, CRN-K1, and experimental data from ion-implanted $a$ - $\mathrm{Si}$ (courtesy of $\mathrm{H}$. Laaziri et $a l^{3}{ }^{3}$ ). Each trace is offset by 2.0 from the one below.

have only one instance of each model, so none of the quantities reported here are ensemble averaged. As a result, they show a degree of statistical noise associated with the system size.

\section{A. Short range order}

The pair correlation function $g_{2}(r)$, which is related to the Fourier transform of the structure factor measured by diffraction, is a basic structural characteristic of an amorphous material model. Figure 1 shows $g_{2}(r)$ for PC-K2, PC$\mathrm{K} 1.5$, PC-K1EDIP2, CRN-K1, and $g_{2}(r)$ derived from x-ray diffraction measurements on ion-implanted $a-\mathrm{Si}^{3}{ }^{3} g_{2}(r)$ for the models is calculated without simulated experimental broadening out to one half the model supercell size. The heights of the first peak in $g_{2}(r)$ for all the models are given in Table I. $g_{2}(r)$ for CRN-K1 and PC-K1EDIP2 is a good match for the experimental $g_{2}(r)$. The effect of the paracrystalline grains becomes more pronounced in PC-K1.5, and

TABLE I. Various measures of short range order in the models as-generated: the average bond angle $\bar{\theta}$, the bond angle standard deviation $\Delta \theta$, and $D_{n}$, the percentage of atoms with first-neighbor coordination $n$.

\begin{tabular}{lcccccccccc}
\hline \hline Model & $\bar{\theta}(\mathrm{deg})$ & $\Delta \theta(\mathrm{deg})$ & $D_{0}$ & $D_{1}$ & $D_{2}$ & $D_{3}$ & $D_{4}$ & $D_{5}$ & $D_{6}$ & $g_{2}(r) 1^{\text {st }}$ peak $^{\mathrm{a}}$ \\
\hline CRN-K1 & 108.1 & 16.1 & 0.0 & 0.2 & 2.9 & 17.1 & 68.7 & 10.9 & 0.2 & 8.0 \\
PC-K1 & 108.3 & 13.8 & 0.0 & 0.8 & 0.8 & 7.4 & 80.1 & 10.9 & 0.0 & 8.7 \\
PC-K1.5 & 108.9 & 13.2 & 0.1 & 0.0 & 0.7 & 4.6 & 84.7 & 9.7 & 0.1 & 9.3 \\
PC-K2 & 108.8 & 11.9 & 0.2 & 0.1 & 1.2 & 8.8 & 84.2 & 5.5 & 0.0 & 11.5 \\
PC-K1EDIP1 & 109.0 & 12.0 & 0.0 & 0.0 & 0.0 & 1.0 & 95.7 & 3.3 & 0.0 & 13.3 \\
PC-K1EDIP2 & 109.0 & 12.0 & 0.0 & 0.0 & 0.0 & 0.0 & 96.2 & 3.8 & 0.0 & 13.5 \\
\hline \hline
\end{tabular}

${ }^{a}$ The height of the first peak in the experimental $g_{2}(r)$ is 6.7 (See Ref. 3). 
especially PC-K2, with the appearance of additional peaks at about $4.5,6.7$, and $10.3 \AA$, which are not present in the experimental $g_{2}(r)$. The peak at $4.5 \AA$ corresponds to the vector $\left\langle\frac{1}{4} \frac{1}{4} \frac{\overline{3}}{4}\right\rangle$ in the crystal, which is the third neighbor distance. We note that the experimental $g_{2}(r)$ shows a weak shoulder at this distance which is not present in the $g_{2}(r)$ of CRN-K1. The peak at $6.7 \AA$ corresponds to $\left\langle\frac{1}{2} \frac{1}{2} 1\right\rangle$, which is the sixth neighbor. Several crystalline pair spacings fall within the width of the peak at $10.3 \AA$, so it is difficult to assign the peak to one predominating crystal vector. The small peak between the first two in CRN-K1, PC-K1.5, and PC-K2 is a well-known defect of models synthesized by quenching from the melt with the SW potential, ${ }^{28}$ and is not present in the EDIP models. $g_{2}(r)$ for PC-K1 and PCK1EDIP1 is indistinguishable from PC-K1EDIP2 and are not shown.

The other common diagnostics of short-range order are the average $\mathrm{Si}-\mathrm{Si}-\mathrm{Si}$ bond angle $\bar{\theta}$, the bond angle standard deviation $\Delta \theta$, and the first-neighbor coordination number distribution. These quantities are shown in Table I. Atoms are considered bonded if they are within $2.7 \AA$ of one another. This bond length was chosen to give the highest percentage of four-coordinated atoms and simultaneously the lowest percentage of five-coordinated atoms. The values of $\Delta \theta$ from $11.9^{\circ}$ to $16.1^{\circ}$ fall within the range for various $a-\mathrm{Si}$ films measured by Raman spectroscopy. ${ }^{29}$ None of the SW models approach the almost-perfect fourfold coordination of the real material, which as mentioned above, reflects the synthesis by quenching from the melt with the SW potential. The high density of coordination defects, particularly overcoordination, also explains the deviation of $\bar{\theta}$ from the tetrahedral value of $109.47^{\circ}$. By contrast, models prepared with EDIP exhibit much better coordination with very few threefold and fivefold coordinated atoms, $\bar{\theta}$ being closer to the tetrahedral angle, and a smaller $\Delta \theta$ than PC-K1, the corresponding SW model.

\section{B. Medium range order}

To characterize MRO, we need information beyond that available from the spherically averaged $g_{2}(r)$. The fourbody correlation function probed by fluctuation microscopy contains such information, ${ }^{16}$ so calculations of the fluctuation microscopy signal from the models provide information about their MRO, as well as a direct comparison to experiment. In fluctuation microscopy, we compute the image intensity variance $V$ of images formed from diffracted radiation as a function of two parameters: $Q$ and $k$. $Q$ controls the length scale at which the technique looks for MRO $(=0.61 / Q) . k$ controls the particular pair spacing $(\propto 1 / k)$ we probe within the sample. Varying $Q$ at constant $k$ is variable resolution microscopy, which yields a length scale $\Lambda$ characteristic of the MRO in the material. Varying $k$ at constant $Q$ is variable coherence microscopy, which yields information about the interior structure of any ordered clusters that exist on the length scale set by $Q$. By choosing a deliberately low microscope resolution, we can probe atomic correlations within volumes $\sim 10 \AA$ across. A large variance with a strong $k$ and $Q$ dependence is a signature of MRO, and a small,

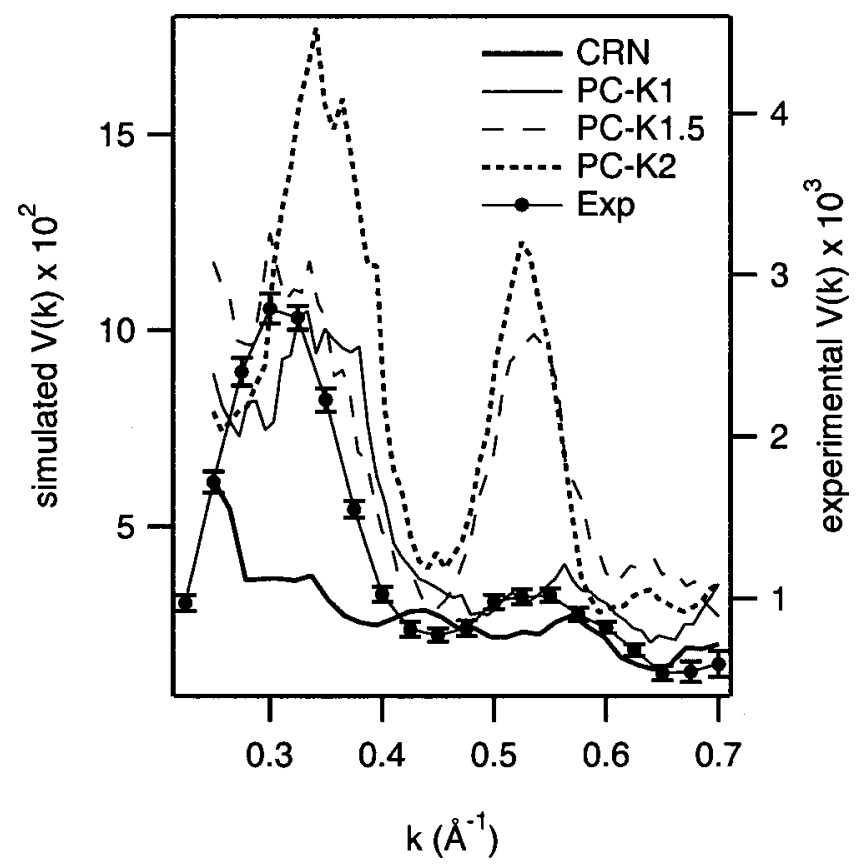

FIG. 2. Variable coherence microscopy simulations for the SW models and experimental data for a sputtered $a$-Si thin film. The simulations refer to the left axis, the data to the right axis. CRN-K1 shows the low variance and lack of structure typical of little or no MRO. The PC models show peaks which increase with increasing seed crystal size.

relatively $k$ and $Q$ independent variance is a signature of structures with little or no MRO, such as a CRN. ${ }^{16}$

Figure 2 shows the calculated variable coherence image variance $V(k)$ for each SW model at a resolution $0.61 / Q$ $=15 \AA$ and experimental data for a sputtered $a$-Si thin film at the same resolution. These simulations carefully follow the imaging process in the electron microscope, including the effects of lens aberrations. MRO is characterized by the position and height of the peaks in $V(k)$. The peaks in $V(k)$ are positioned near the diffraction maxima ${ }^{30}$ for $a-\mathrm{Si}$ at $k$ $=0.31 \AA^{-1}$ and $k=0.56 \AA^{-1} .{ }^{1}$ The peak heights, and therefore the degree of MRO, increase monotonically with increasing seed crystal size for the SW PC models. $V(k)$ for CRN-K1 is representative of other CRN models ${ }^{13}$ and lacks a strong signature of MRO. Figure 2 also illustrates the high sensitivity of fluctuation microscopy to MRO compared to the relatively MRO-insensitive diffraction techniques (see Fig. 1).

Figure 3 shows the calculated $V(k)$ for PC-K1, PCK1EDIP1, and PC-K1EDIP2. PC-K1EDIP1, which was prepared with the same seed geometry as PC-K1, shows only hints of paracrystallinity in its $V(k)$. PC-K1EDIP2, which had twice as many atoms in the initial seeds, clearly shows the double-peak pattern of paracrystallinity. This shows that, while it is possible to synthesize a PC model with both the SW potential and EDIP, the EDIP synthesis preserves less of the initial crystalline order than the SW synthesis. Also, there is no correlation between $V(k)$ and the coordination defect density across potentials, so this measure of PC MRO is relatively insensitive to the potential-dependent details of the defect structure of the models. 


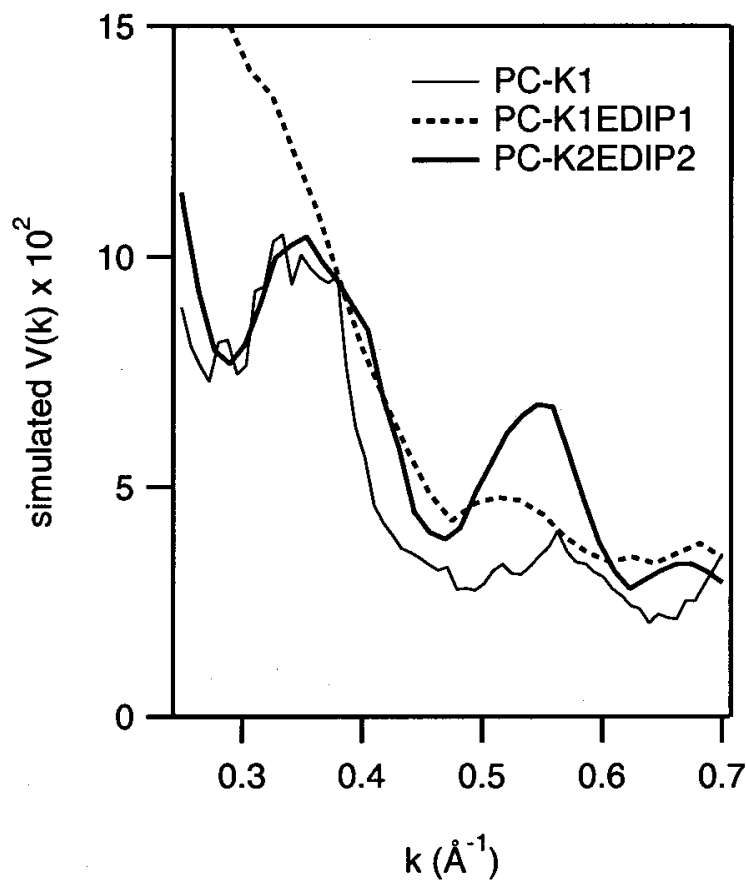

FIG. 3. Variable coherence microscopy simulations for PC-K1, PCK1EDIP1, and PC-K1EDIP2. PC-K1EDIP1 shows little medium range order, whereas PC-K1EDIP2 has clear peaks at the same positions as PC-K1.

The position of the peaks in $V(k)$ for the PC models compares favorably with the experiment. However, the absolute magnitude of the calculated variance for all models is much larger than that observed in the experiment. This discrepancy is, at least partially, likely due to the relatively small size of these models which will artificially enhance the calculated image variance. Preliminary simulations on larger models show a reduced variance, but significantly more modeling effort is needed to achieve more quantitative agreement with experiment. To compare the models to the experiment despite this difference in magnitude, we have calculated the ratio of $V(k)$ at the first peak to $V(k)$ at the second peak, as shown in Table II. Only PC-K1 and PC-K1EDIP1 are close to the experimental value of 2.7. The other PC models and CRN-K1 have second peaks that are too large in comparison to the first.

Direct information on the MRO length scale is provided by the variable resolution variance $V(Q)$. Analysis of $V(Q)$ calculated $^{15,16}$ at $k=0.35 \AA^{-1}$ yields the MRO length scale $\Lambda$, which is given for all models in Table II. As expected, $\Lambda$ is related to the size of the seed crystals and increases monotonically in the SW models from PC-K1 to PC-K2. PCK1EDIP2 has a slightly smaller $\Lambda$ than that of PC-K1 (SW) model, while PC-K1EDIP1 has a larger $\Lambda$ than either PC-K1 or PC-K1EDIP2. No variable resolution microscopy experimental data are vailable at this point for comparison.

Also shown in Table II is the $g_{2}(r)$ correlation length $L_{c}$, which is the length beyond which there are no more structural oscillations in $g_{2}(r)$. Since $L_{c}$ is derived from $g_{2}(r)$, there is no difference in $L_{c}$ between CRN-K1 and PC-K1. $L_{c}$ does increase with increasing seed crystal size in the SW PC models.

A more intuitive measure of MRO is the distribution of dihedral angles $P(\phi)$. The dihedral angle $\phi$ is the angle of rotation of two tetrahedra about the bond that connects them, so it involves fourth neighbor atoms and is a physically intuitive segment of the parameter space of the four-body correlation function probed by fluctuation microscopy. Diamond-like crystalline $\mathrm{Si}$ is characterized by $\phi=60^{\circ}$, called staggered bonding, while a hexagonal lonsdalelite-like structure has $\phi=0^{\circ}$, called eclipsed bonding. Zachariasen originally proposed the $\mathrm{CRN}$ model as a structure with a constant $P(\phi) .{ }^{11}$

There are effects clearly attributable to MRO from the PC grains in $P(\phi)$. Figure 4 shows $P(\phi)$ for the fourcoordinated atoms in the SW PC models (so as to avoid problems defining the angle for miscoordinated atoms). The PC models show increasing weight of staggered bonds $(\phi$ $\sim 60^{\circ}$ ) compared to CRN-K1 with increasing seed crystal size, while CRN-K1 shows a preference for eclipsed bonding $\left(\phi \sim 0^{\circ}\right)$. Considering spatial correlations between dihedral angles is one way to tell if the increase is really due to the residual effects of the grains. Following Luedtke and Landman, ${ }^{24}$ we calculate the sum and difference correlation functions $C_{s}\left(\phi_{1}+\phi_{2}\right)$ and $C_{d}\left(\phi_{1}-\phi_{2}\right)$ between adjacent, connected dihedral angles. If the dihedral angles had no spatial correlations, we could calculate $C_{s}\left(\phi_{1}+\phi_{2}\right)$ and $C_{d}\left(\phi_{1}-\phi_{2}\right)$ from

$$
C_{s}\left(\phi_{1}+\phi_{2}\right)=\int_{0}^{60^{\circ}} P\left(\phi^{\prime}\right) P\left(\left(\phi_{1}+\phi_{2}\right)-\phi^{\prime}\right) d \phi^{\prime}
$$

TABLE II. Various measures of MRO in the models as-generated: the variable coherence peak hight ratio $V\left(k \simeq 0.3 \AA^{-1}\right) / V\left(k \simeq 0.55 \AA^{-1}\right)$, the variable resolution microscopy correlation length $\Lambda$ at $k=0.35 \AA^{-1}$, the $g_{2}(r)$ correlation length $L_{c}$, the seed crystal diameter $d_{\text {seed }}$, the percentage of atoms in the topologically crystalline (TC) grains \% TC, the number of topologically crystalline grains $N_{g}$, and their average diameter $d_{\mathrm{PC}}$. All distances are in $\AA$.

\begin{tabular}{lccccccc}
\hline \hline Model & $V(k)$ ratio $^{\mathrm{a}}$ & $\Lambda$ & $L_{c}^{\mathrm{b}}$ & $d_{\text {seed }}$ & $\%$ TC & $N_{g}$ & $d_{\mathrm{PC}}$ \\
\hline CRN-K1 & 1.4 & $4.62 \pm 0.06$ & 7 & - & 0 & 0 & - \\
PC-K1 & 2.6 & $7.8 \pm 0.2$ & 7 & 11.1 & 13 & 2 & 11.4 \\
PC-K1.5 & 1.2 & $8.5 \pm 0.2$ & 9.5 & 12.3 & 32 & 2 & 16.9 \\
PC-K2 & 1.4 & $15.4 \pm 0.6$ & 12 & 14.0 & 57 & 4 & 18.3 \\
PC-K1EDIP1 & 2.8 & $10.1 \pm 0.6$ & 7.0 & 10.8 & 10 & 2 & 10.0 \\
PC-K1EDIP2 & 1.5 & $7.1 \pm 0.9$ & 10.2 & 13.6 & 15 & 3 & 10.1 \\
\hline \hline
\end{tabular}

${ }^{\text {aT }}$ The experimental $V(k)$ ratio for sputtered $a$-Si is 2.7 .

${ }^{\text {b}}$ The uncertainty in $L_{c}$ is $\pm 1 \AA$. 


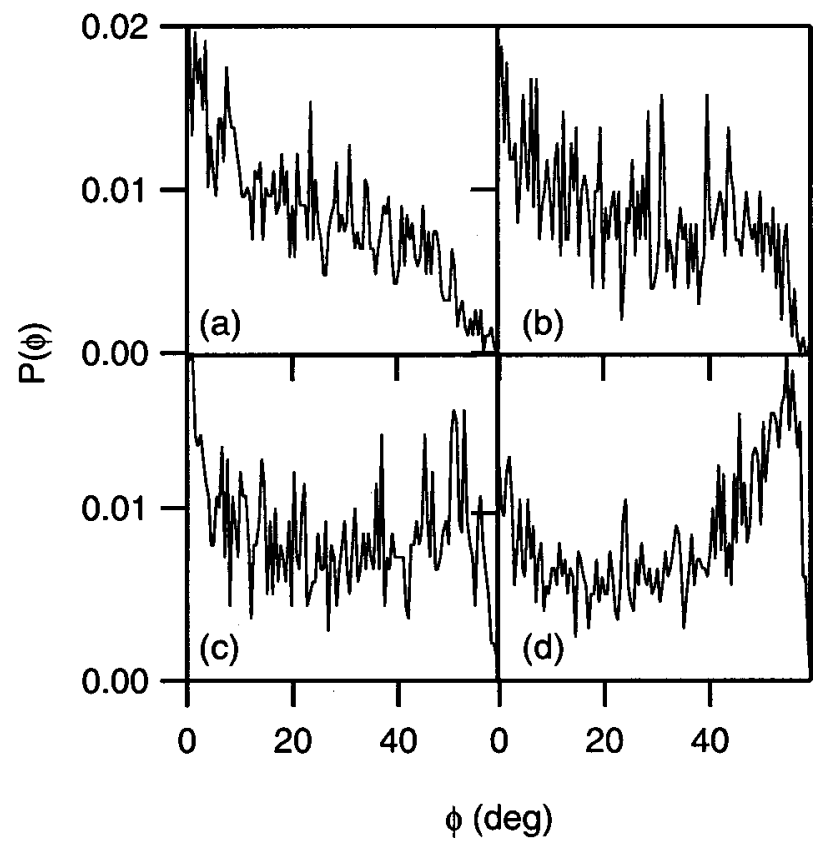

FIG. 4. The dihedral angle distribution $P(\phi)$ for: (a) CRN-K1, (b) PC-K1, (c) PC-K1.5, and (d) PC-K2. The increasing MRO is reflected in the height of the peak at $\phi \sim 60^{\circ}$.

and

$$
C_{d}\left(\phi_{1}-\phi_{2}\right)=\int_{0}^{60^{\circ}} P\left(\phi^{\prime}\right) P\left(\left(\phi_{1}-\phi_{2}\right)+\phi^{\prime}\right) d \phi^{\prime} .
$$

The model dihedral correlation functions and the calculations for uncorrelated dihedrals are shown in Figs. 5 and 6 for the SW models. The degree of correlation, as measured by the

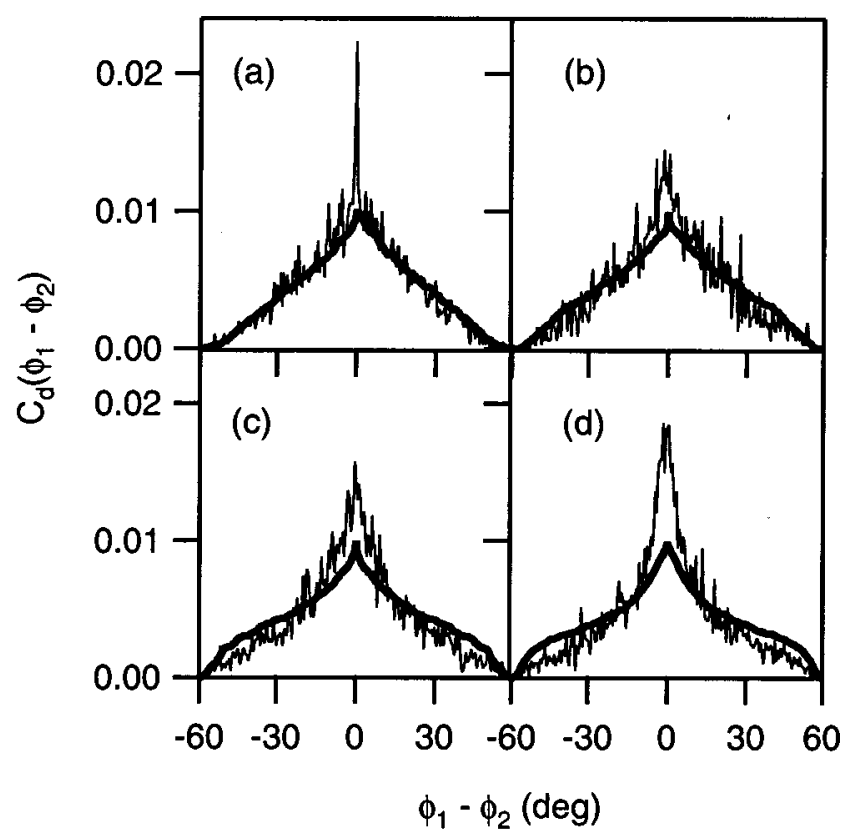

FIG. 5. Dihedral difference correlation function $C_{d}\left(\phi_{1}-\phi_{2}\right)$ for: (a) CRNK1, (b) PC-K1, (c) PC-K1.5, and (d) PC-K2. The light line is the calculated value and the dark line is the expectation for uncorrelated dihedral angles calculated from Eq. (2).

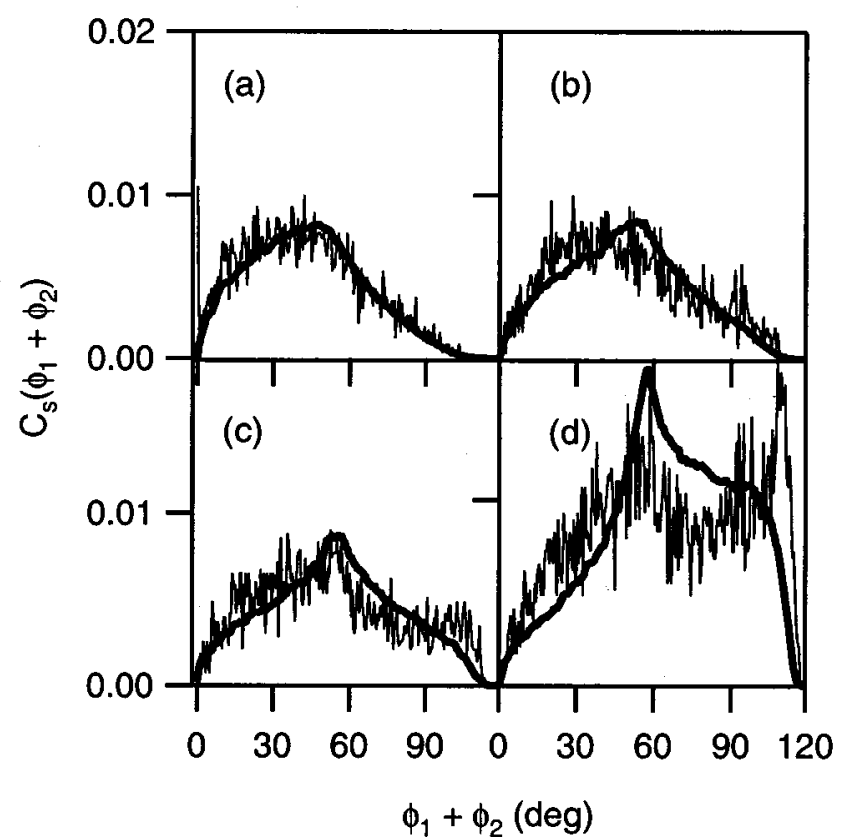

FIG. 6. Dihedral sum correlation function $C_{s}\left(\phi_{1}+\phi_{2}\right)$ for: (a) CRN-K1, (b) PC-K1, (c) PC-K1.5, and (d) PC-K2. The light line is the calculated value and the dark line is the expectation for uncorrelated dihedral angles calculated from Eq. (1).

integrated absolute difference between the model correlation functions and the prediction for a random distribution of dihedral angles, increases monotonically with seed crystal size for both $C_{s}\left(\phi_{1}+\phi_{2}\right)$ and $C_{d}\left(\phi_{1}-\phi_{2}\right)$. The peaks above the prediction for uncorrelated dihedrals in $C_{d}\left(\phi_{1}-\phi_{2}\right)$ at $\phi_{1}$ $-\phi_{2}=0^{\circ}$ (Fig. 5) shows that like dihedral angles tend to cluster together, and that more clustering takes place with increasing seed-crystal size. The peaks in $C_{s}\left(\phi_{1}+\phi_{2}\right)$ near $120^{\circ}$ (Fig. 6) show that the clusters are likely to be made up of tetrahedra in the staggered configuration of a Si crystal.

The dihedral angles remain somewhat equivocal, however. The peak at $50^{\circ}$ in $C_{s}\left(\phi_{1}+\phi_{2}\right)$ indicates some correlation between staggered and eclipsed dihedral angles. Also, CRN-K1 exhibits significant incidence of and correlation between eclipsed dihedral angles, evidenced by the peaks at $0^{\circ}$ in Figs. 4(a) and 6(a).

To further characterize the nature of the MRO, we analyzed the topology of the bond networks. Topological measures are particularly useful for the analysis of amorphous model structures because they are relatively insensitive to the effects of strain. Table III summarizes the ring statistics of

TABLE III. Ring statistics for the models as-generated.

\begin{tabular}{lccccccc}
\hline \hline & \multicolumn{7}{c}{ \% of rings with $N$ members } \\
\cline { 2 - 8 } Model & 3 & 4 & \multicolumn{1}{c}{5} & \multicolumn{1}{c}{6} & \multicolumn{1}{c}{7} & \multicolumn{1}{c}{8} & 9 \\
\hline CRN-K1 & 0.2 & 1.5 & 30.8 & 40.1 & 14.1 & 3.6 & 3.9 \\
PC-K1 & 0.0 & 1.4 & 33.8 & 48.2 & 9.5 & 2.8 & 1.4 \\
PC-K1.5 & 0.0 & 1.4 & 29.7 & 54.7 & 8.1 & 1.3 & 0.7 \\
PC-K2 & 0.1 & 0.8 & 18.9 & 66.7 & 8.3 & 1.7 & 1.3 \\
PC-K1EDIP1 & 0.4 & 2.2 & 25.3 & 58.2 & 12.3 & 1.7 & 0.1 \\
PC-K1EDIP2 & 0.5 & 1.4 & 25.6 & 61.5 & 10.4 & 0.7 & 0.0 \\
\hline \hline
\end{tabular}


the models. We define a ring as the shortest closed path of bonds leaving an atom along one bond and returning on another that encloses no shorter paths back to the original atom. ${ }^{31}$ Diamond-like crystalline Si has only six-membered rings. The percentage of six rings in the SW models increases with increasing seed crystal size at the expense of five and seven rings, consistent with a trend toward crystalline Si bonding. However, PC-K1EDIP1 and PC-K1EDIP2 show a higher percentage of six rings than PC-K1, which would seem to indicate a greater degree of crystallinity.

We have also employed a more informative topological tool, the Schläfli cluster. ${ }^{19,32}$ The Schläfli cluster of an atom is defined as those atoms and bonds involved in the rings emanating from the central atom. A Schläfli cluster is identified by its Schläfli circuit symbol ${ }^{33}$ and the number of atoms in the cluster. All known small-unit-cell four-connected crystals have uniquely labeled Schläfli clusters in this scheme $^{34,35}$ For example, diamond-like crystalline silicon has the Schläfli cluster $6_{2} \cdot 6_{2} \cdot 6_{2} \cdot 6_{2} \cdot 6_{2} \cdot 6_{2}: 29$, indicating that there are two different six-membered rings emanating from each pair of bonds of the central atom and that there are 29 atoms in the cluster. A four-connected crystal with hexagonal stacking (the lonsdalelite structure), has the same $6_{2} \cdot 6_{2} \cdot 6_{2} \cdot 6_{2} \cdot 6_{2} \cdot 6_{2}$ circuit symbol but contains only 27 atoms. We therefore refer to an atom which has the Schläfli cluster characteristic of a crystal as "topologically crystalline." We apply this tool to search for MRO by computing the Schläfli cluster for every atom in a model, then searching for the $6_{2} \cdot 6_{2} \cdot 6_{2} \cdot 6_{2} \cdot 6_{2} \cdot 6_{2}: 29$ cluster characteristic of crystalline Si. The Si Schläfli cluster itself has a diameter of $9 \AA$, which is a convenient MRO length scale, and connected regions of Si Schläfli clusters set a MRO length scale intrinsic to the model. We call such connected regions of Si Schläfli clusters the "paracrystalline grains" in the PC models.

Figure 7 shows for each of the SW PC models only those atoms which are inside the PC grains. No CRN model that we have examined has even one Si Schläfli cluster. ${ }^{19}$ PC-K2 has topologically crystalline grains at the position of all four of the seed crystals. In PC-K1 and PC-K1.5, only two of the four seed crystals remain topologically crystalline; the other two have dissolved back into the disordered matrix. This implies the existence of some stability condition for the existence of the grains depending on their size, density, and processing history, which will be the subject of future simulations. The EDIP models show similar behavior, with PCK1EDIP1 also retaining two grains. The number and average diameter of the PC grains $d_{\mathrm{PC}}$ and the percent of the atoms in each model within the PC grains may be found in Table II. PC-K1EDIP1 has fewer atoms in smaller PC grains that PC$\mathrm{K} 1$, but has a larger variable resolution length $\Lambda$. This may be due to subtle increased order within the PC grains in PC-K1EDIP1. The magnitude of the peaks in $V(k)$ shows a correlation with the topologically crystalline fraction across all the models.

As has been briefly noted previously, ${ }^{19}$ the PC grains, which we identify solely by their topology, show greater order than the disordered matrix by spatial measures as well. Figure 8 shows the bond angle and dihedral angle distributions of the SW PC models broken into contributions from
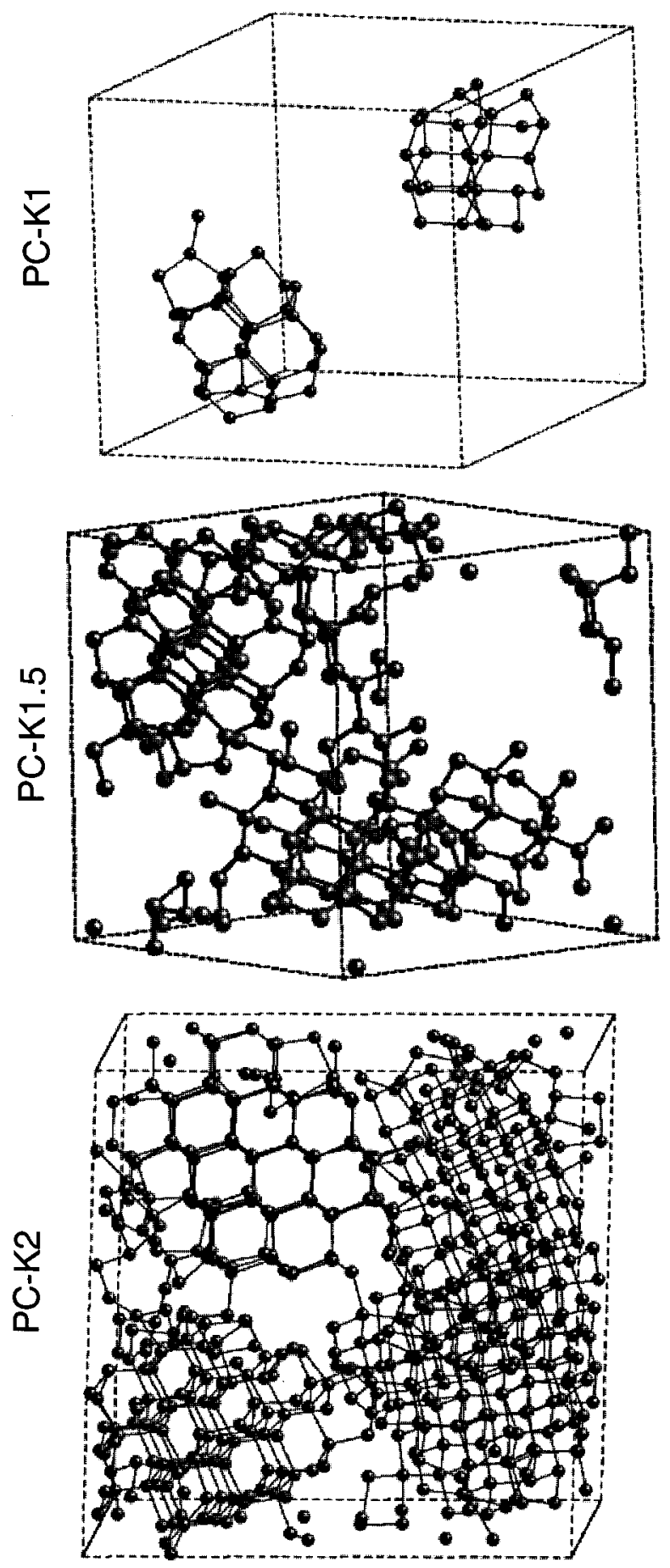

FIG. 7. A ball-and-stick representation of only those atoms in the PC SW models that participate in the $\operatorname{Si} 6_{2} \cdot 6_{2} \cdot 6_{2} \cdot 6_{2} \cdot 6_{2} \cdot 6_{2}: 29$ Schläfli clusters. These are the topologically crystalline paracrystalline grains.

the atoms in the PC grains and the atoms outside the PC grains. Bonds across the boundary are ignored, so the two $P(\phi)$ curves for each model in Fig. 8 will not quite sum up to the corresponding $P(\phi)$ curve in Fig. 4 . The bond angle distributions of the PC grains are narrower than those of the intergranular matrix, and the features in $P(\theta)$ near $60^{\circ}$ are only seen in the intergranular matrix. As shown in Table IV, $\Delta \theta$ is reduced inside the grains, both compared with the intergranular material and the entire model (see Table I), and $\bar{\theta}$ 


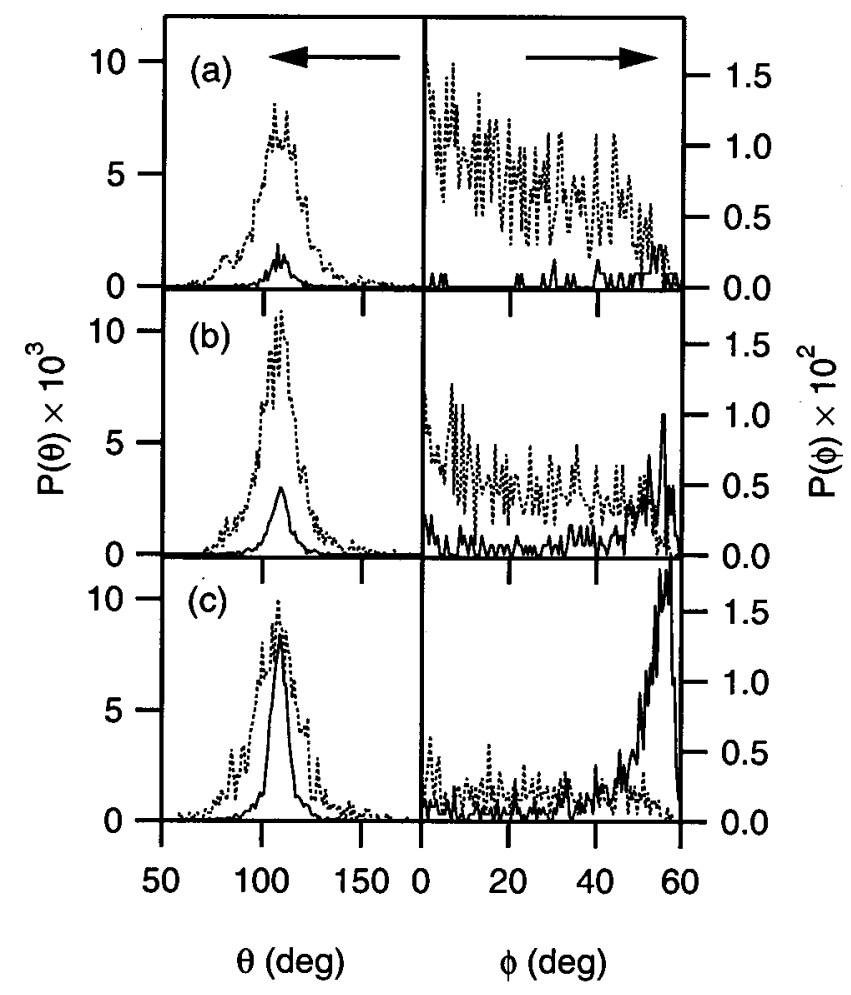

FIG. 8. Partial $P(\theta)$ and $P(\phi)$ computed for those atoms in the PC grains (solid line) and those in the intergranular matrix (dashed line) for: (a) PC$\mathrm{K} 1$, (b) PC-K1.5, and (c) PC-K2.

moves closer to the tetrahedral angle. The grains also exhibit the staggered bonding characteristic of the crystal almost exclusively, while the intergranular material has a $P(\phi)$ similar to CRN-K1 [Fig. 4(a)]. The ordering within the grains is not surprising, since the topology constrains the real-space structure, but it has important implications for the physical properties, particularly the Raman spectra (see Sec. IV C).

The PC grains in the SW models are also characterized by a lower local energy density as measured by the bonding energy assigned by the SW potential to individual atoms in the PC grains versus those in the intergranular material. Table IV reports the average difference from the whole model average energy for the atoms in these two regions. The energy difference is small, but the grains are very small and even atoms in the center of the grains are very close to the intergranular boundaries.

TABLE IV. Energy and bond angle characteristics of the PC grains in the SW models. $\bar{\theta}_{\mathrm{PC}}$ and $\Delta \theta_{\mathrm{PC}}$ are the average bond angle and bond angle standard deviation for the PC grains, respectively, and $\bar{\theta}_{\mathrm{IG}}$ and $\Delta \theta_{\mathrm{IG}}$ are the same quantities for the intergranular material. $U$ is the total energy/atom of the model as assigned by the SW potential, $U_{\mathrm{PC}}$ is the energy/atom of the atoms in the PC grains minus $U$, and $U_{\mathrm{IG}}$ is the energy/atom of the atoms not in the PC grains minus $U$. Energies are in $\mathrm{eV}$, angles in degrees.

\begin{tabular}{lccccccc}
\hline \hline Model & $\bar{\theta}_{\mathrm{PC}}$ & $\Delta \theta_{\mathrm{PC}}$ & $\bar{\theta}_{\mathrm{IG}}$ & $\Delta \theta_{\mathrm{IG}}$ & $U^{\mathrm{a}}$ & $U_{\mathrm{PC}}$ & $U_{\mathrm{IG}}$ \\
\hline PC-K1 & 108.6 & 6.3 & 108.1 & 14.7 & -4.16 & -0.07 & 0.01 \\
PC-K1.5 & 109.1 & 7.8 & 108.4 & 12.5 & -4.17 & -0.04 & 0.02 \\
PC-K2 & 109.3 & 7.9 & 107.9 & 14.7 & -4.18 & -0.07 & 0.06 \\
\hline
\end{tabular}

${ }^{\mathrm{a}} U$ for $\mathrm{CRN}-\mathrm{K} 1$ is $-4.16 \mathrm{eV}$.
In summary, in this section we have provided various structural and topological analyses of the PC models with an emphasis on MRO. We have discussed several characterization techniques and their usefulness for probing MRO. We have shown that the PC models show qualitative agreement with fluctuation microscopy data, which is not shown by CRN models. The models cannot yet give a quantitative representation of the experimental structures. Comparison of models prepared with the SW potential and EDIP suggests that EDIP produces smaller grains with a higher degree of internal order. This comparison also shows that the nature of medium range order is related mainly to the degree of remaining crystallinity, rather than to nature and density of structural defects. In the following sections we analyze the physical properties of the models related to atomic vibrations and electronic behavior.

\section{VIBRATIONAL PROPERTIES}

Now we turn our attention to the vibrational properties of the models. We calculate the VDOS, characterize the localization, stretching/bending character, and acoustic/optic character of the vibrational modes, then consider the Raman spectra of the models and the partial Raman spectra of the PC grains. In this section we consider only the SW PC models and PC-K1EDIP1. Marinov and Zotov ${ }^{36}$ have characterized a 216-atom CRN model created with the Wooten, Winer, and Weaire (WWW) bond-switching algorithm ${ }^{37}$ using the same techniques used here, and we do not expect CRN-K1 to show any significant differences. PC-K1EDIP1 is studied to investigate differences created by changing potentials; since PC-K1EDIP2 has no SW analog, it is not studied in this section.

\section{A. Calculation procedures}

To calculate the vibrational properties, we use a Kirkwood-type potential, ${ }^{27}$ which is especially suited for vibrational studies of covalently bonded materials. We first performed a structural relaxation of all the PC models using the Kirkwood potential. The equilibrium $\mathrm{Si}-\mathrm{Si}$ bond length was set to the experimentally observed $\mathrm{Si}-\mathrm{Si}$ distance ${ }^{3,38}$ of $2.36 \AA$ and the equilibrium $\mathrm{Si}-\mathrm{Si}-\mathrm{Si}$ bond angle was set to the ideal tetrahedral value of $109.47^{\circ}$. The strain energy was minimized by an iterative process in which each atom was displaced one at a time in the direction of the force without allowing bond breaking or reconstruction. This process was repeated until convergence was achieved. The average shift of the atoms in the relaxed models varies from $0.10 \AA$ for PC-K1EDIP1 to $0.20 \AA$ for PC-K2. This relaxation significantly reduced the short-range disorder of the models. The total strain energy $E_{\text {tot }}$ as given by the Kirkwood potential, the strain energy per $\mathrm{Si}$ atom $E_{\text {tot }} / N$, the bond angle standard deviation $\Delta \theta$, and the bond length standard deviation $\Delta r$ of the relaxed models are given in Table $\mathrm{V}$. The reduced shortrange disorder is evident in the reduced $\Delta \theta$ (see Table I). As noted in Sec. I, this relaxation does not remove the PC character of the models.

All eigenfrequencies and eigenvectors were determined by direct diagonalization of the full dynamical matrix as given by the Kirkwood potential. These values were used to 
TABLE V. Various measurements of short range order in the PC models after relaxation with the Kirkwood potential. $E_{\text {tot }}$ is the total strain energy from the Kirkwood potential, $E_{\text {tot }} / N$ is the strain energy/atom, $\Delta \theta$ is the bond angle standard deviation, and $\Delta r$ is bond length standard deviation.

\begin{tabular}{lcccc}
\hline \hline Model & $E_{\text {tot }}(\mathrm{eV})$ & $E_{\text {tot }} / N(\mathrm{eV})$ & $\Delta \theta(\mathrm{deg})$ & $\Delta r(\AA)$ \\
\hline PC-K1 & 193.93 & 0.3788 & 7.60 & 0.032 \\
PC-K1.5 & 228.00 & 0.3262 & 7.11 & 0.035 \\
PC-K2 & 297.43 & 0.2974 & 6.52 & 0.032 \\
PC-K1EDIP1 & 107.12 & 0.2096 & 7.50 & 0.031 \\
\hline \hline
\end{tabular}

calculate the VDOS. The values of the stretching and bending force constants used for the energy relaxation and the VDOS calculations are 125 and $5 \mathrm{~N} / \mathrm{m}$, respectively; they were adjusted previously ${ }^{36}$ to reproduce approximately the position of the main vibrational bands of $a$-Si. To analyze the character of the vibrational modes we calculate the participation ratio $p_{c}(\omega)$, the stretching character $S(\omega)$, and the total phase quotient $q_{\Sigma}(\omega)$. Definitions and a detailed discussion of these parameters are given elsewhere. ${ }^{36}$

For the calculation of the first-order Raman spectra of the models we use the bond polarizability approximation. ${ }^{36,39,40}$ Each Raman line is additionally broadened by $15 \mathrm{~cm}^{-1}$ to account for the additional disorder not present in the computer generated models and their finite size. This broadening was determined by matching the calculated width of the TO Raman line after broadening to the experimental value. The partial Raman spectra of the PC grains were calculated using the atomic displacements obtained by projecting the contribution of these atoms and their nearest neighbors out of the dynamical matrix.

\section{B. Vibrational modes}

The general character of the VDOS, shown in Fig. 9, is similar in all the PC models and is in good agreement with

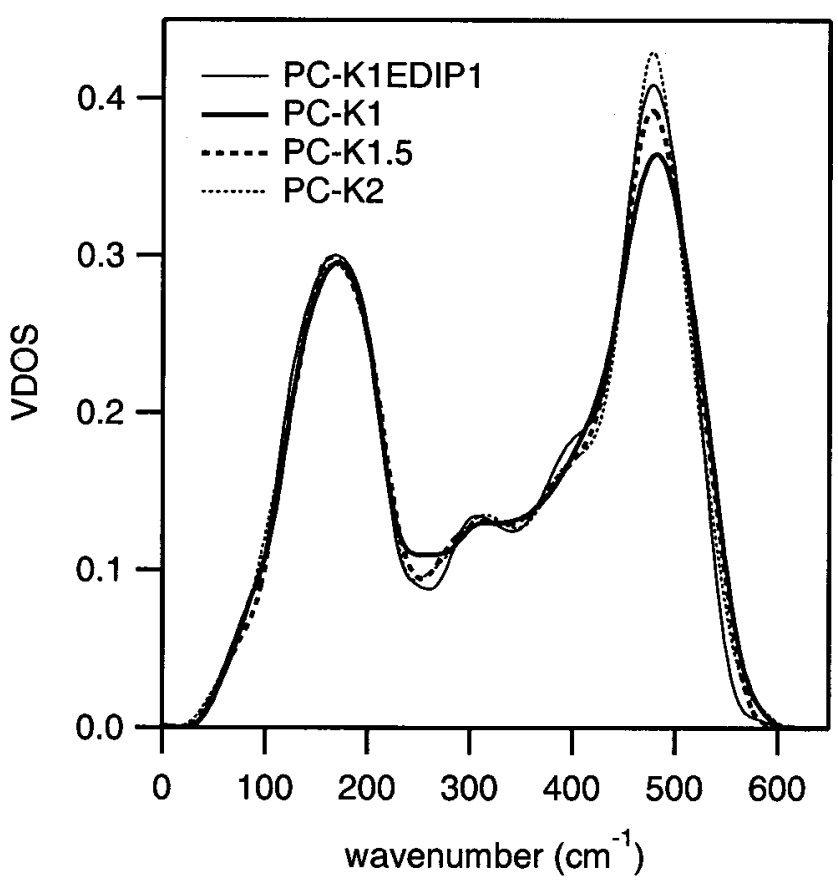

FIG. 9. The vibrational density of states of the PC models.

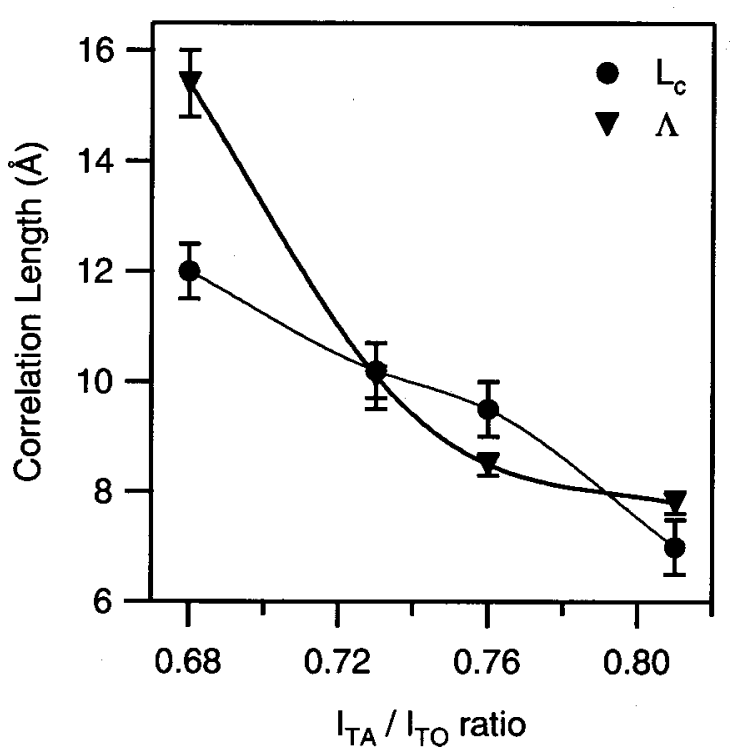

FIG. 10. MRO correlation lengths from variable resolution microscopy $(\Lambda)$ and $g_{2}(r)\left(L_{c}\right)$ as a function of the $I_{\mathrm{TA}} / I_{\mathrm{TO}}$ ratio of the VDOS. The lines are a guide to the eye.

experimental neutron scattering measurements. ${ }^{7,8}$ The calculated VDOS also compares well with previous VDOS calculations for $a$-Si models constructed by different methods using various potentials. ${ }^{36,41-46}$ As in crystalline $\mathrm{Si}$, the peak at $\sim 175 \mathrm{~cm}^{-1}$ is commonly assigned to transverse acoustic (TA)-like vibrations, the weak peak at $\sim 325 \mathrm{~cm}^{-1}$ to longitudinal acoustic (LA)-like vibrations, the weak shoulder at $\sim 400 \mathrm{~cm}^{-1}$ to longitudinal optic (LO)-like vibrations, and the high-frequency peak at $\sim 500 \mathrm{~cm}^{-1}$ to transverse optic (TO)-like vibrations. In the SW models, the TA peak practically does not change position with increasing PC MRO, while the TO peak shifts to slightly lower frequencies in PC-K2. The LA and LO peaks also become slightly more pronounced. The VDOS for PC-K1EDIP1 is similar to that for PC-K1, but the TO peak is higher, and the LO/LA peaks are better resolved. As we see below, this is related to the improved short-range order and lower defect density of the EDIP model.

The most significant change in the VDOS is the change in the ratio of the intensities of the TA and TO peaks $I_{\mathrm{TA}} / I_{\mathrm{TO}}$. Figure 10 shows that various MRO correlation lengths decrease with increasing $I_{\mathrm{TA}} / I_{\mathrm{TO}}$ ratio. This confirms by direct simulation that the $I_{\mathrm{TA}} / I_{\mathrm{TO}}$ intensity ratio in the VDOS can be a sensitive probe of the degree of MRO. Of the models considered, only $\mathrm{PC}-\mathrm{K} 1$ has a $I_{\mathrm{TA}} / I_{\mathrm{TO}}$ ratio close to the experimental value of 0.88 obtained from inelastic neutron scattering experiments, ${ }^{8}$ which agrees with the variable coherence microscopy simulations presented in Sec. III B.

The participation ratio $p_{c}(\omega)$ for the PC models is shown in Fig. 11; $p_{c} \omega$ of PC-K1EDIP1 is generally similar to that of PC-K1 and is not shown. $p_{c}(\omega)$ measures the localization of the vibrational modes; $p_{c}(\omega)$ close to 1 indicates a delocalized mode, while $p_{c}(\omega)$ close to 0 indicates a localized mode. The delocalized modes in all three models have $p_{c}(\omega)$ of $\sim 0.58$, despite the different sizes of the mod- 


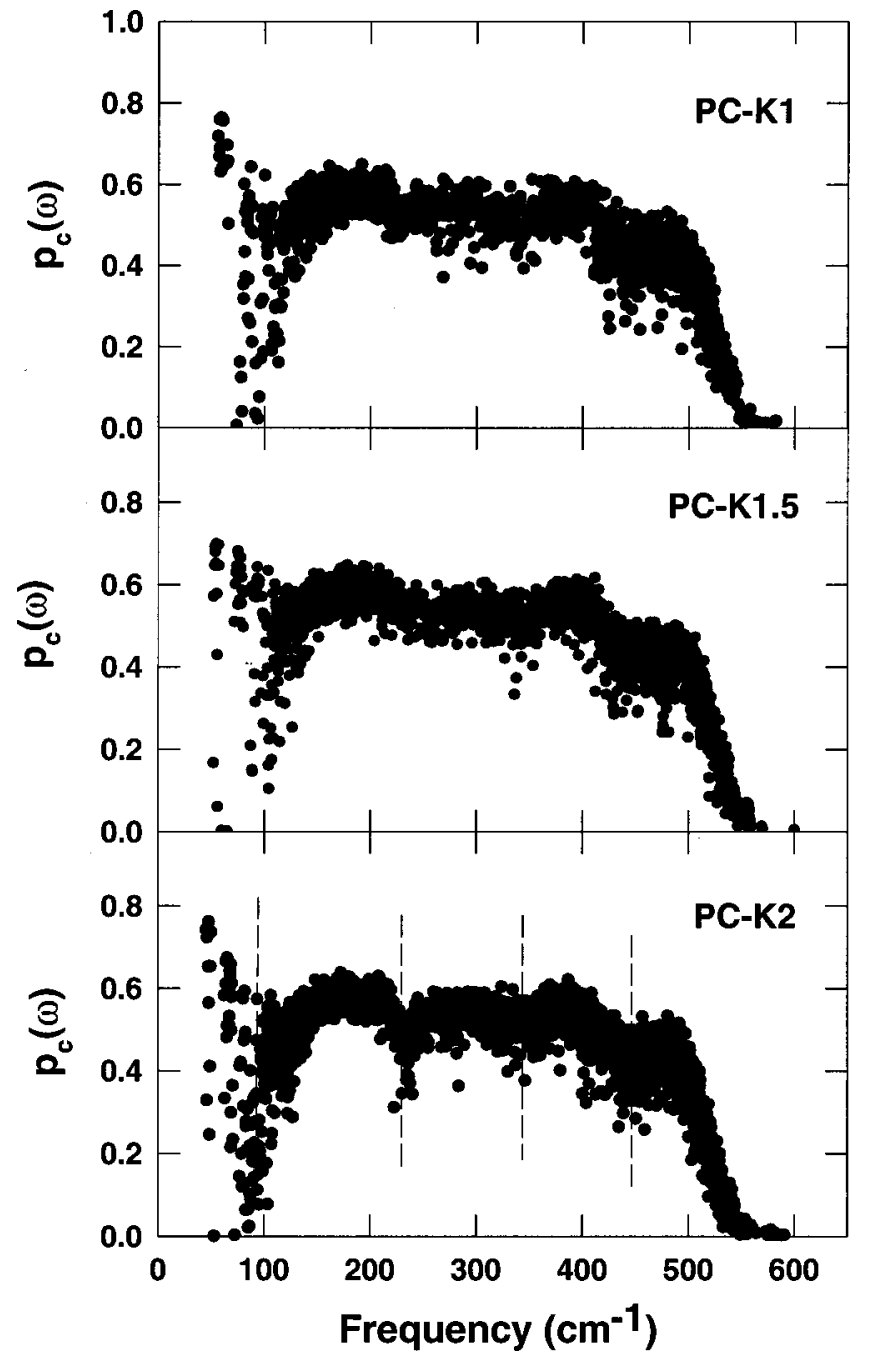

FIG. 11. The participation ratio $p_{c}(\omega)$ of the vibrational modes of the SW PC models. The vertical dashed lines mark the position of the band edges.

els. The localized modes are grouped around several frequencies: $90,230,340,450$, and $550 \mathrm{~cm}^{-1}$, marked by the vertical dashed lines in Fig. 11. By comparison with the position of the main peaks in the VDOS (Fig. 9), we see that these frequencies mark the position of the band edges. The low-frequency TA modes are localized on $D_{1}, D_{2}$, and $D_{3}$ atoms, which have small residual strain energy as given by the Kirkwood potential. $\left(D_{n}\right.$ denotes an atom with coordination number $n$.) The number of strongly localized modes $\left(p_{c}(\omega)<0.2\right)$ increases quadratically with the concentration of $D_{1}+D_{2}+D_{3}$ atoms. PC-K1EDIP1, which has no $D_{1}$ or $D_{2}$ defects, exhibits a much higher $p_{c}(\omega)$ at the $90 \mathrm{~cm}^{-1}$ band edge. The main difference in the participation ratio between the PC models and the WWW CRN model ${ }^{37}$ as well as $a$-Si models generated by the activation-relaxation technique (ART), ${ }^{47}$ investigated previously, ${ }^{36,48}$ is that the CRNs do not show low-frequency localization. However, CRN models of $a$-Si produced with the Biswas, Grest, and Soukoulis method ${ }^{42}$ also exhibit low-frequency localization on undercoordinated atoms, which implies that such features are not specific to the PC structure, or the SW potential or EDIP.

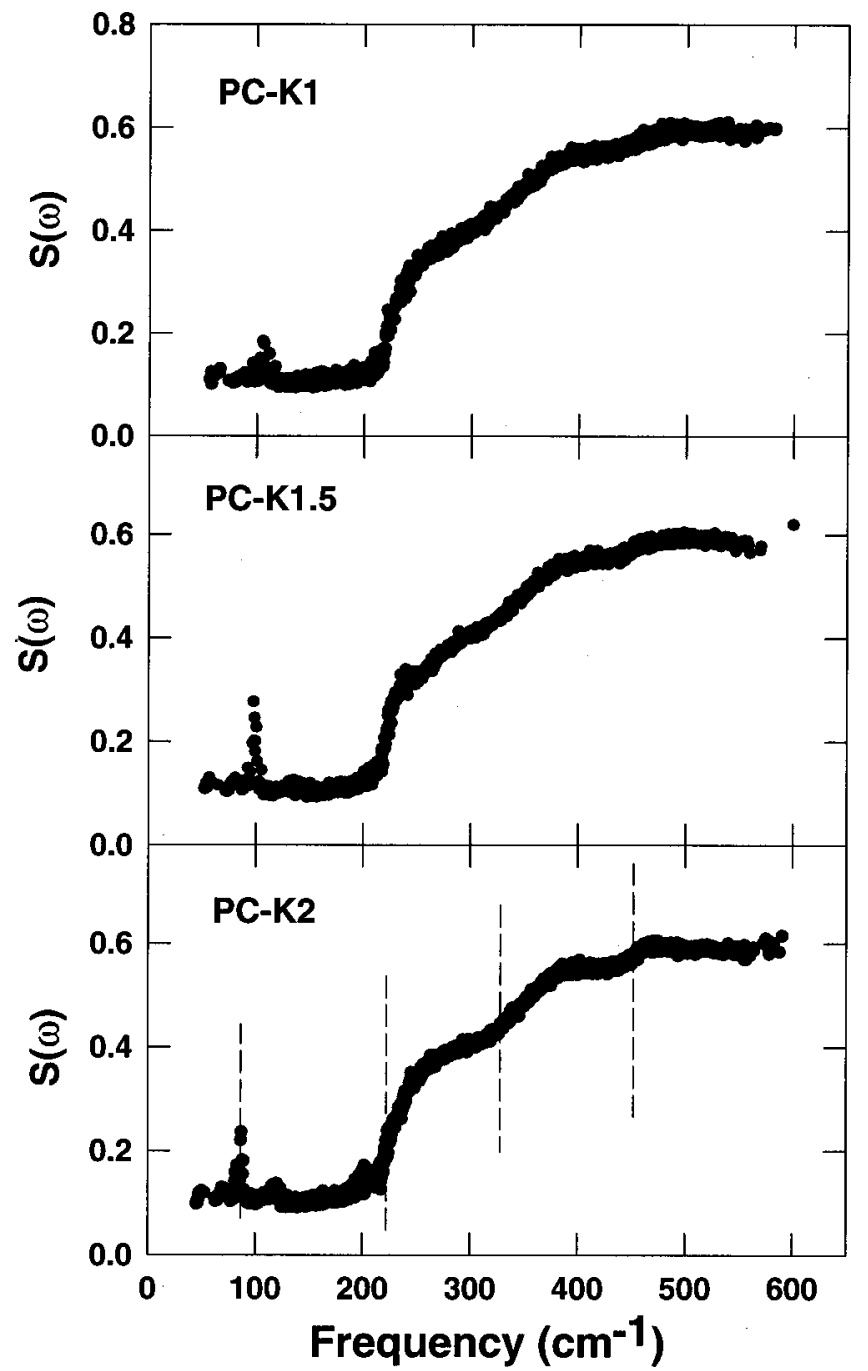

FIG. 12. The stretching character $S(\omega)$ of the vibrational modes of the SW PC models. The vertical dashed lines mark the position of the band edges.

The modes near the $220-240$ and $330-360 \mathrm{~cm}^{-1}$ band edges are predominantly localized on atoms which are at the boundary of the PC grains. These atoms are either surface atoms of the grains and have one or more bonds to $\mathrm{Si}$ atoms outside the grain or vice versa- $\mathrm{Si}$ atoms which have one or more nearest neighbors belonging to the PC grains. Generally, these are $\mathrm{Si}$ atoms with moderate residual strain energy. The mid-frequency localization is less pronounced in PC-K1, which reflects the smaller number and size of the PC grains in this model, but also suggests a more relaxed grain boundary region. In addition, there are a few modes near these band edges in PC-K1.5 and PC-K2 which are strongly localized on $D_{5}$ atoms or on four-coordinated $\mathrm{Si}$ atoms with large bond-angle distortions. Clearly, the heterogeneous structure of these models, with PC grains separated by a disordered matrix, leads to pinning of the vibrations in the grain boundary region.

The high-frequency modes are localized on $D_{5}$ and $D_{6}$ overcoordinated atoms or atoms with very large residual strain energy. This effect is further enhanced by the fact that the $D_{5}$ atoms themselves have very large strain energy. They 


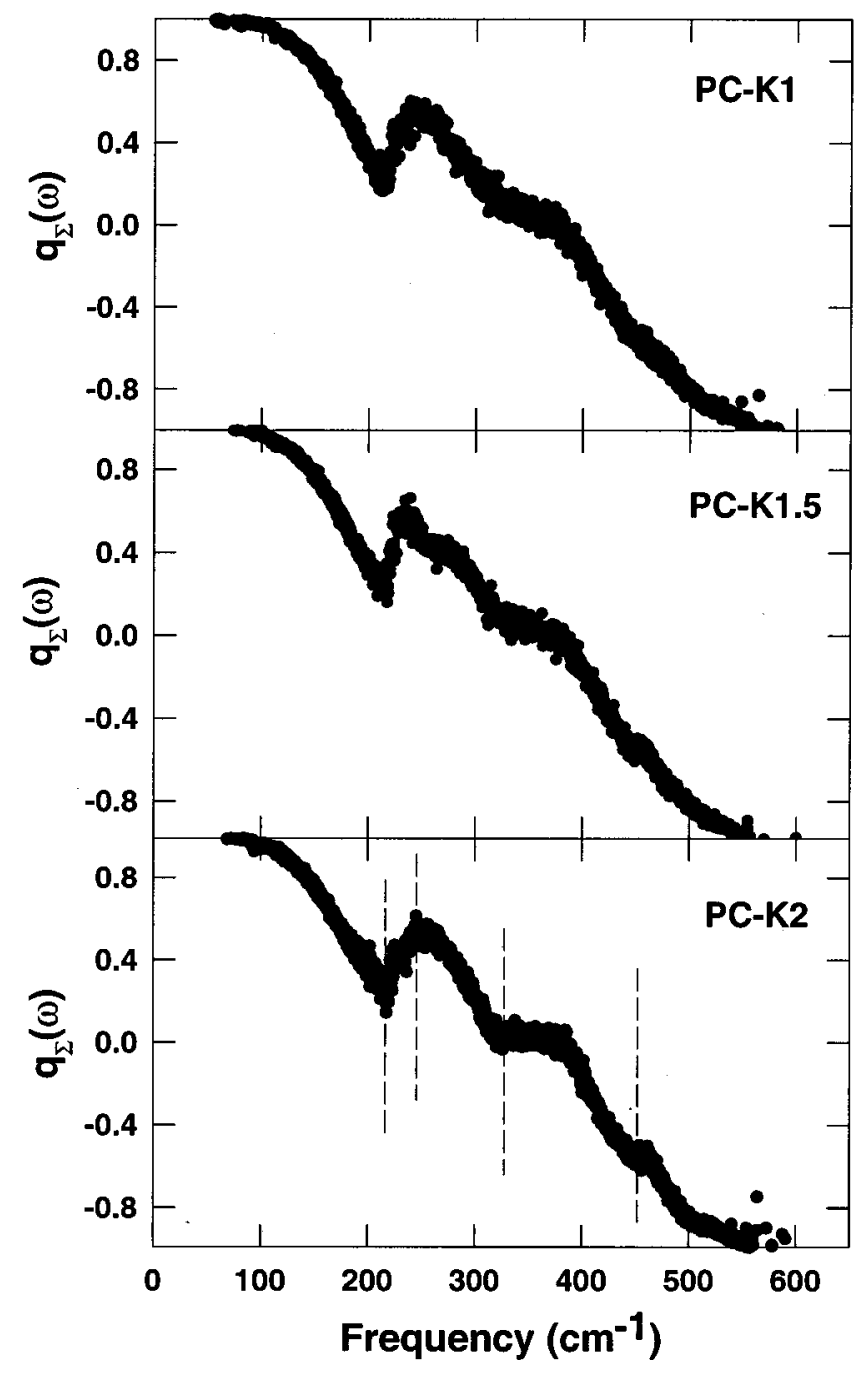

FIG. 13. The phase quotient $q_{\Sigma}(\omega)$ of the vibrational modes of the SW PC models. The vertical dashed lines mark the position of the band edges.

are always within the first $15 \%$ of the atoms with the highest strain energy.

The stretching character $S(\omega)$ for the SW PC models is shown in Fig. 12. $S(\omega)$ is near 1 if the mode is predominantly of the bond-stretching type and near 0 if the mode is predominantly of the bond-bending type. The character of $S(\omega)$ is generally the same for the models and is similar to $S(\omega)$ for other $a$-Si models. ${ }^{36,46}$ The low-frequency TA modes below $230 \mathrm{~cm}^{-1}$ are predominantly bending in character. The modes between 230 and $450 \mathrm{~cm}^{-1}$ (LA and LO modes) are mixed bending + stretching in character. The high-frequency TO modes above $450 \mathrm{~cm}^{-1}$ are predominantly stretching in character. With increasing PC MRO the fine structure of the stretching character in the mid-frequency region (LA and LO bands) increases significantly. Unlike in CRN models ${ }^{36}$ of $a$-Si, the PC models show no gap between the low- and mid-frequency modes. The presence of defect $\mathrm{Si}$ atoms leads to this increased mixing of the TA and LA modes, so PC-K1EDIP1 (not shown) has a much smaller density of modes in the gap region. Several low-frequency modes with much higher stretching character $(S(\omega)>0.2)$ are observed at $\sim 80-105 \mathrm{~cm}^{-1}$. They are located predomi- nantly on $D_{3}$ atoms. Such modes are not observed in CRN models generated by the $\mathrm{WWW}^{36}$ or $\mathrm{ART}^{48}$ methods.

The general character of the total phase quotient $q_{\Sigma}(\omega)$, shown in Fig. 13, is also similar in all the SW PC models and PC-K1EDIP1. $q_{\Sigma}(\omega)$ measures the correlations between the individual atomic displacements. If $q_{\Sigma}(\omega)$ is near 1 , the mode is acoustic-like, and if $q_{\Sigma}(\omega)$ is near -1 , the mode is optic-like. $q_{\Sigma}(\omega)$ also exhibits singularities near the band edges at $\sim 230, \sim 320$, and $\sim 450 \mathrm{~cm}^{-1}$. The modes below $230 \mathrm{~cm}^{-1}$ are acoustic-like, and the modes between 250 and $320 \mathrm{~cm}^{-1}$ are mixed acoustic +optic. The modes between 320 and $450 \mathrm{~cm}^{-1}$ are mixed optic + acoustic. Finally, the high-frequency modes beyond $450 \mathrm{~cm}^{-1}$ are optic-like in character. The calculated phase quotients confirm the assignment of the 175 and $500 \mathrm{~cm}^{-1}$ bands as acoustic and optic bands and show that this character is preserved in PC models of $a$-Si. The fine structure of the phase quotient near the band edges becomes more pronounced with increasing PC $\mathrm{MRO}$ and a new singularity develops at $\sim 380 \mathrm{~cm}^{-1}$. Unlike the SW PC models, in the WWW model of $a$-Si there are no modes in the vibrational gap between 230 and $250 \mathrm{~cm}^{-1} .36$ The number of such modes is reduced in PC-K1EDIP1. In the SW PC models the modes in that frequency range are predominantly localized on $D_{1}, D_{2}$, and $D_{3}$ atoms, as we have seen from an analysis of the participation ratio.

\section{Polarized Raman spectra}

The total reduced VV and VH polarized Raman spectra for the SW PC models and PC-K1EDIP1 are shown in Fig. 14. The spectra are scaled to the same number of Si atoms. In the VH spectra, the LA-LO range is practically featureless. The TO peak increases in intensity, becomes narrower, and shifts to slightly higher frequencies with increasing PC MRO in the SW models. The high-frequency TO modes are predominantly of stretching character (see Fig. 12) and thus they are very sensitive to tetrahedral disorder. The average bond-length disorder in the four models is similar (see Table $\mathrm{V})$, but the bond angle standard deviation $\Delta \theta$ decreases with increasing PC MRO in the SW models. With increasing $\Delta \theta$, the TO peak intensity falls and its full width at half maximum $\Gamma_{\text {TO }}$ grows, as shown in Table VI. This trend is borne out in PC-K1EDIP1, which has a smaller $\Delta \theta$ than PC-K1 and a higher $I_{\mathrm{TO}}$. The calculated $\Gamma_{\mathrm{TO}}$ using the empirical correlation proposed by Beeman et al. ${ }^{9}\left(\Gamma_{\mathrm{TO}}=15+6 \Delta \theta\right)$, also shown in Table VI, is in excellent agreement with $\Gamma_{\text {то }}$ determined from the simulated spectra using $15 \mathrm{~cm}^{-1}$ uniform broadening.

The behavior of the VV polarized spectra is the same as the $\mathrm{VH}$ spectra because the average depolarization ratio $\rho$ $=I_{\mathrm{VH}} / I_{\mathrm{VV}}$ is the same in all models $(\langle\rho\rangle=0.57)$. Since, however, the VV spectra are much more sensitive to the details of the structure, we observe a weak LA peak near 300 $\mathrm{cm}^{-1}$ in the VV spectrum of PC-K1.

Similar to the VDOS, the $I_{\mathrm{TA}} / I_{\mathrm{TO}}$ ratio of the Raman spectra has been proposed as a measure of MRO. ${ }^{10}$ As observed in the experiment, the calculated intensity ratio $I_{\mathrm{TA}} / I_{\mathrm{TO}}$ for the $\mathrm{VV}$ polarized spectra is slightly larger than $I_{\mathrm{TA}} / I_{\mathrm{TO}}$ for the $\mathrm{VH}$ polarized spectra in all three models. 

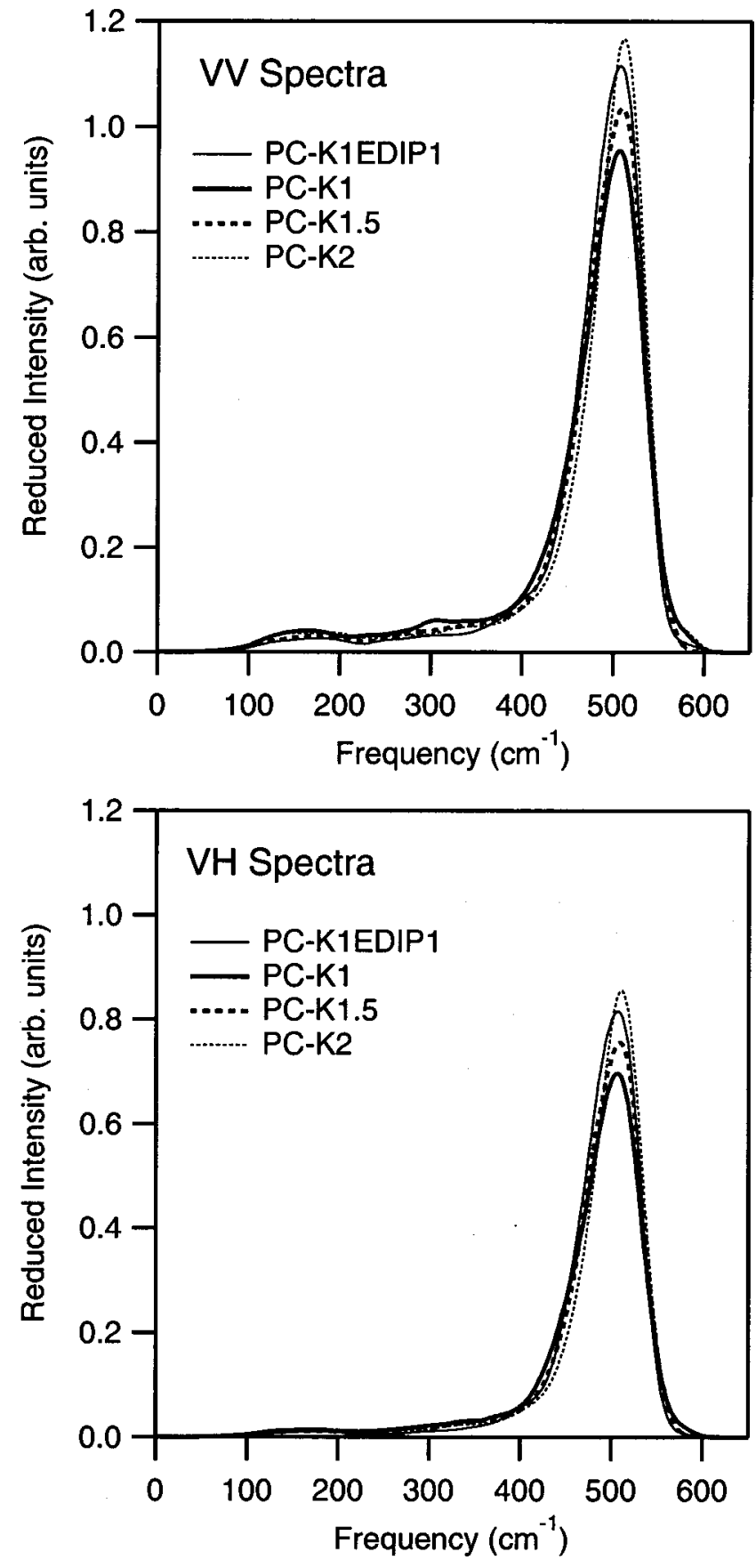

FIG. 14. The VV and VH polarized Raman spectra of the PC models.

$I_{\mathrm{TA}} / I_{\mathrm{TO}}$ for both the $\mathrm{VV}$ and $\mathrm{VH}$ spectra behave similarly with increasing size of the models. For simplicity, we will consider further only the $\mathrm{VV}$ spectra $I_{\mathrm{TA}} / I_{\mathrm{TO}}$ in the SW models.

The VV spectrum $I_{\mathrm{TA}} / I_{\mathrm{TO}}$ ratio is $\sim 0.043$ for $\mathrm{PC}-\mathrm{K} 1$, $\sim 0.023$ for PC-K1EDIP1, and reduces to $\sim 0.031 \pm 0.001$ for PC-K1.5 and PC-K2. However, the correlation with PC MRO is less pronounced than the correlation of the $I_{\mathrm{TA}} / I_{\mathrm{TO}}$ ratio from the VDOS (see Figs. 9 and 10). Generally, the decrease of $I_{\mathrm{TA}} / I_{\mathrm{TO}}$ is consistent with the increase of the size of the PC grains, taking into account that for crystalline $\mathrm{Si}$ $I_{\mathrm{TA}} / I_{\mathrm{TO}}$ should be zero.
TABLE VI. Characteristics of the reduced VH polarized Raman spectra of the PC models. $w_{\mathrm{TO}}$ is the TO mode frequency, $I_{\mathrm{TO}}$ is its intensity, and $\Gamma_{\mathrm{TO}}$ is its width.

\begin{tabular}{lcccc}
\hline \hline Model & $\omega_{\mathrm{TO}}\left(\mathrm{cm}^{-1}\right)$ & $I_{\mathrm{TO}}$ & $\Gamma_{\mathrm{TO}}\left(\mathrm{cm}^{-1}\right)$ & $\Gamma_{\mathrm{TO}}{ }^{\mathrm{a}}\left(\mathrm{cm}^{-1}\right)$ \\
\hline PC-K1 & 507 & 0.70 & 59 & 60.6 \\
PC-K1.5 & 508 & 0.76 & 58 & 57.7 \\
PC-K2 & 510 & 0.86 & 54 & 54.1 \\
PC-K1EDIP1 & 506 & 0.81 & 66 & 60.0 \\
\hline \hline
\end{tabular}

${ }^{\mathrm{a}}$ Calculated from the empirical relation $\Gamma_{\mathrm{TO}}=15+6 \Delta \theta$ (see Table V) proposed by Beeman et al. ${ }^{9}$

Comparing the calculated $I_{\mathrm{TA}} / I_{\mathrm{TO}}$ values with intensity ratios determined from published polarized spectra of rfsputtered (RFS) and chemical-vapor deposited (CVD) $a$-Si, we see first that the model intensity ratios are smaller than the experimental ones. $I_{\mathrm{TA}} / I_{\mathrm{TO}}=0.10-0.12$ for typical RFS $a-\mathrm{Si}$, and $I_{\mathrm{TA}} / I_{\mathrm{TO}}=0.068-0.078$ for CVD $a-\mathrm{Si}{ }^{49,50} \mathrm{The}$ calculated $I_{\mathrm{TA}} / I_{\mathrm{TO}}$ values are closer to the experimental $I_{\mathrm{TA}} / I_{\mathrm{TO}}$ values for CVD $a$-Si samples, which generally have a more ordered structure. It is also interesting to note that the experimental $I_{\mathrm{TA}} / I_{\mathrm{TO}}$ values decrease with increasing plate temperature in RFS samples and with increasing substrate temperature in CVD samples. Evidently, the relaxation of the $a$-Si structure at higher temperatures leads to smaller local strains and correspondingly smaller $I_{\mathrm{TA}} / I_{\mathrm{TO}}$ values. Altogether, these results suggest that the PC-K1.5 and PC-K2 models have more ordered structure than typical RFS and CVD $a$-Si samples prepared at relatively low substrate temperatures, so PC-K1 is again the most physically realistic of the current models.

Simulations of the partial Raman spectra (PRS) of the PC grains in the SW models are shown in Fig. 15. The PRS are scaled to the same number of $\mathrm{Si}$ atoms in the grains. Unlike the total polarized spectra, the PRS show significant differences from one model to another. The differences are especially pronounced in the $\mathrm{VV}$ spectra. $I_{\mathrm{TA}} / I_{\mathrm{TO}}$ decreases from 0.32 for PC-K1, to 0.24 in PC-K1.5, to 0.11 in PC-K2. This indicates increasing order within the $\mathrm{PC}$ grains with increasing grain size, as noted in Sec. III B. At the same time the depolarization ratio of the TA band increases from 0.20 in $\mathrm{PC}-\mathrm{K} 1$ to 0.55 in $\mathrm{PC}-\mathrm{K} 2$. This means that in $\mathrm{PC}-\mathrm{K} 2$ the low-frequency TA modes, localized on $\mathrm{Si}$ atoms from the PC grains, become more symmetric bending in nature than in PC-K1. Most significant are the differences in the LA-LO region. The depolarization ratio of the PRS in the LA-LO region is much higher than the average depolarization ratio in the total Raman spectra. The peak at $\sim 300$ $\mathrm{cm}^{-1}$ in the total VV spectrum of PC-K1 (Fig. 14) obviously arises from the corresponding feature in the PRS of the $\mathrm{PC}$ grains in PC-K1. Features similar to those in the LA-LO region of the PRS of PC-K1.5 have been observed experimentally in the VV Raman spectra of $\mathrm{RFS}^{49,50} a$-Si and glow discharge ${ }^{29} a$-Si:H deposited at low substrate temperatures.

The changes in the LA-LO region of the PRS do not correlate with the increasing crystallinity of the PC grains. However, we have shown that the modes at the band edges near 250, 320, and $450 \mathrm{~cm}^{-1}$, which separate the LA and LO 


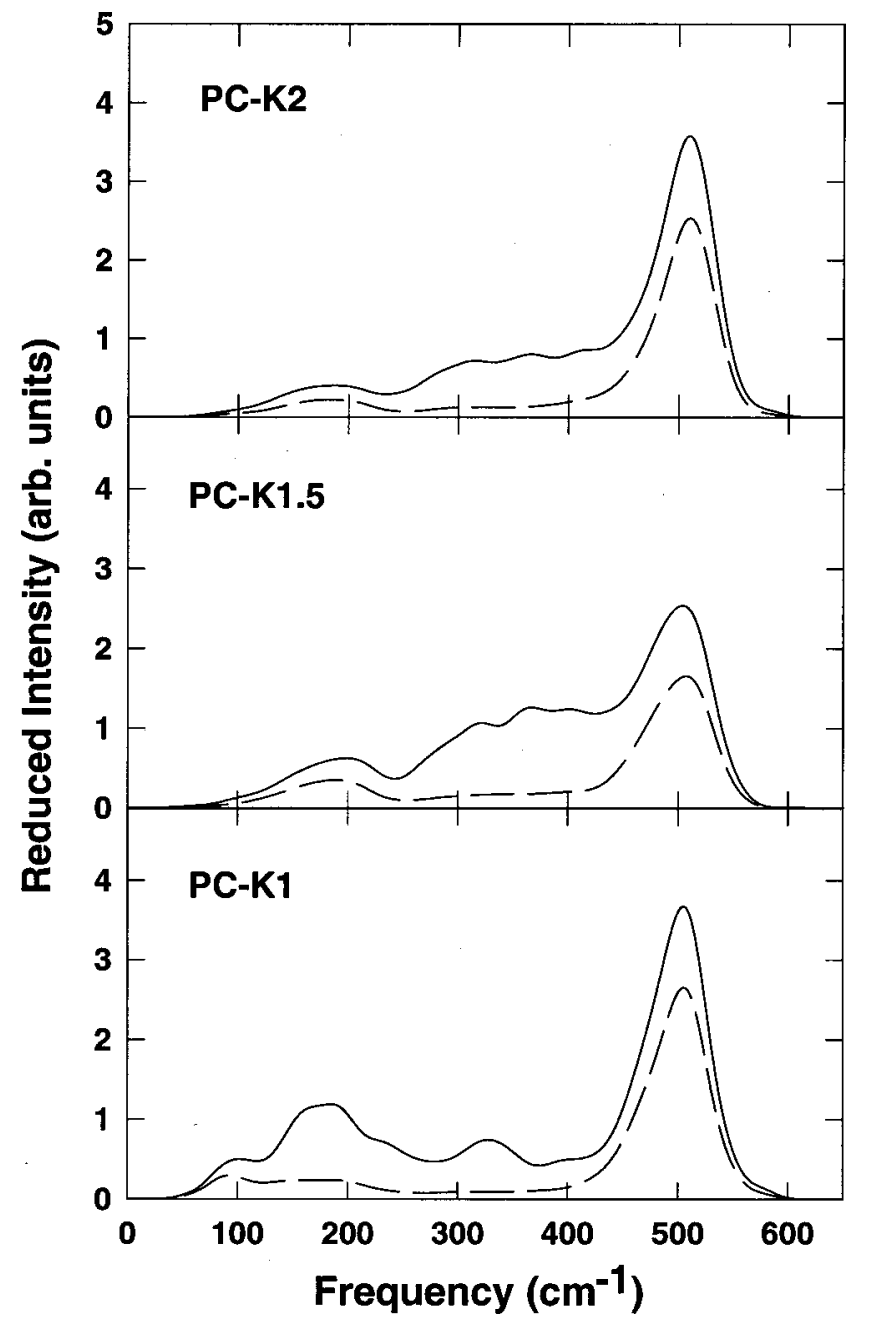

FIG. 15. The VV (solid line) and VH (dashed line) polarized partial Raman spectra of the PC grains in the SW models.

modes, are mostly localized on $\mathrm{Si}$ atoms in the grain boundary regions. This suggests that the differences are probably related to the nature of these grain boundaries. Additional study is required to quantify the changes observed.

\section{ELECTRONIC STRUCTURE}

In this section we present the EDOS for the SW models and PC-K1EDIP1. Prior to our electron properties studies the models generated with Keblinksi et $a .^{22}$ MD program using the SW potential were further relaxed by simulated annealing and quenching with the empirical MD code Estrelle, described elsewhere, ${ }^{51,52}$ which uses EDIP. ${ }^{25}$ For the actual EDOS calculation the Fireball local-basis $a b$ initio MD code developed by Sankey and co-workers ${ }^{53}$ was employed, but only to construct and diagonalize the Hamiltonian matrix for a given configuration of atomic coordinates. To produce the EDOS graphs we used a Gaussian broadened form for $\delta(E$ $-E_{i}$ ).

The EDOS of all the models is presented in Figs. 16 and 17. All the models show the expected features at $-15,-10$, and $-5 \mathrm{eV}$, which are assigned to $s, p$, and $s p^{3}$ states, respectively. As noted in Secs. II and III, all the initial geometries prepared with the SW potential are significantly over-

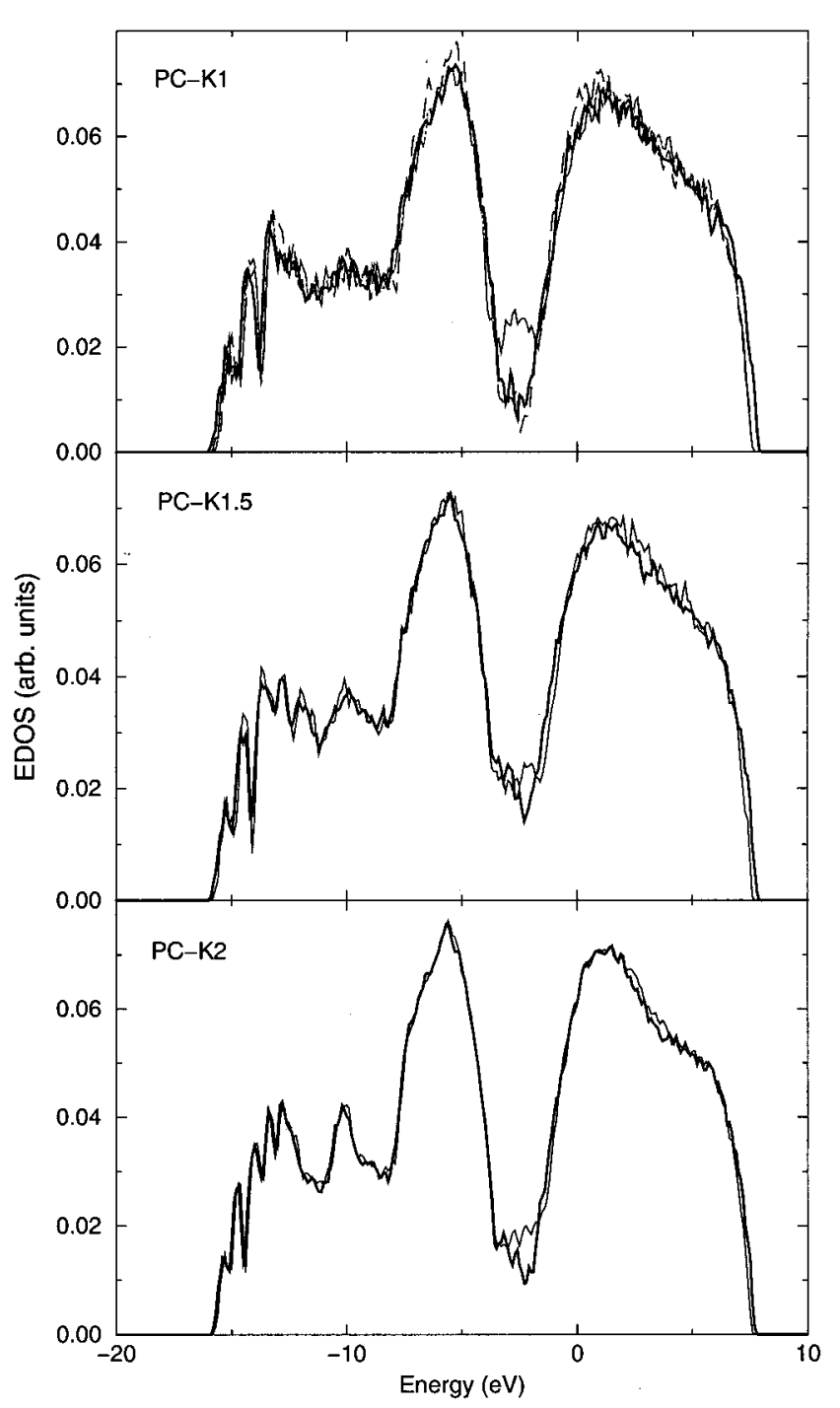

FIG. 16. The EDOS of the PC models. The light solid line is the initial configuration, the heavy solid line is after annealing and quenching with "Estrelle." The dashed line in the top panel is PC-K1EDIP1 in the initial configuration.

coordinated, with an average coordination of 4.15-4.20. This, as we can see from the "unquenched" geometry EDOS data in Fig. 16, produces a much larger density of defect states in the band-gap region than is found in real materials. In an attempt to overcome this difficulty by forcing the models to rearrange and reduce the coordination, each model was annealed with Estrelle at $1000 \mathrm{~K}$ for $20 \mathrm{ps}$. This procedure resulted in a decrease of the average coordination for every model, although the reduction ranged from a significant drop, from 4.20 to 4.07 , for PC-K1 to almost nothing for $\mathrm{PC}-\mathrm{K} 1.5$. This difference is likely due to chance local configurations of atoms which cannot be reconstructed without melting the model and removing its PC character. This is supported by the fact that PC-K1EDIP1 and PC-K1EDIP2 have an average coordination number of about 4.02, which is smaller than any of the structures created with the SW potential. PC-K1 was also quenched directly (without annealing) from its initial configuration with $a b$ initio $\mathrm{MD}$, the 


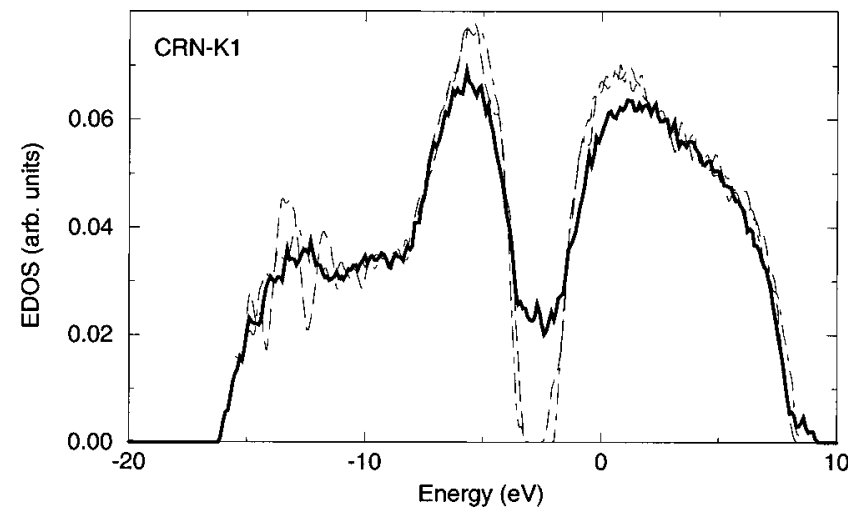

FIG. 17. The EDOS of CRN-K1 after annealing and quenching with "Estrelle" (solid line), a WWW-method CRN (long dashed line), and an ART CRN (Ref. 47) (dot-dashed line).

results of which are very similar to the results of the EDIP anneal and subsequent quench and are not shown.

None of these post-synthesis treatments reduced the average coordination of the SW models to an acceptable level. As we can see from Fig. 16, even PC-K1, for which we were able to achieve the best "improvement" of the electron properties, still has a significant concentration of defect states in the band-gap region, and $\mathrm{CRN}-\mathrm{K} 1$ retains a high density of defects. There is also no obvious connection between the size of the PC grains in a model and the depth of its band gap. There is, however, a transparent connection between the density of geometric defects and the deterioration of the electron properties of the models: the larger the deviation from perfect four fold coordination, the higher the concentration of defect electron states in the band-gap region. This is demonstrated by the clean band gap, shown in Fig. 17, of a perfectly coordinated CRN produced by the WWW method ${ }^{37}$ and an almost perfectly coordinated CRN produced by ART. $^{47}$

Better results are obtained using EDIP during synthesis, as shown by the deeper band gap of PC-K1EDIP1 in the top panel of Fig. 16. The EDOS of this model is as good as that of the best CRN models synthesized by quenching from the melt with EDIP. ${ }^{54}$

\section{CONCLUSIONS}

We have examined the structural, vibrational, and electronic properties of several PC model structures of $a$-Si created by molecular dynamics, with particular attention to MRO. These models were created by molecular dynamics synthesis from the melt in the presence of small seed crystals.

Several characterization methods were found to be effective for describing MRO in models. In general, methods that involve higher-order atom position correlations, such as fluctuation microscopy simulations and the dihedral angle distribution, are sensitive to MRO. The pair correlation function $g_{2}(r)$ available from diffraction is not as sensitive. We also demonstrated that Schläfli cluster topological analysis allows the identification of topologically crystalline regions in models, even if those grains are heavily strained so that they have little or no signature in $g_{2}(r)$. The fluctuation microscopy simulations show strong correlations with other measures of MRO, particularly the fraction of the atoms in the model that are topologically crystalline. Our structural analysis shows post priori that the MRO of the models increases with the size of the seed crystals.

We have shown by direct simulation that the ratio of the transverse acoustic to transverse optic peak intensities $I_{\mathrm{TA}} / I_{\mathrm{TO}}$ in the VDOS, and to a lesser extent in the polarized Raman spectra, can also be a probe of MRO. In addition the singularities in the vibrational characteristics near the band edges and the structure in the LA-LO region in the VDOS become more pronounced with increasing PC MRO. We have also confirmed for PC $\mathrm{Si}$ models the empirical correlation $^{9}$ between the full width at half maximum and the frequency of the TO Raman peak. Taken together, these results suggest that high-resolution inelastic neutron scattering and polarized Raman spectroscopy experiments should be able to provide valuable information about the degree of MRO in real $a$-Si materials and should also be able to distinguish between $\mathrm{Si}$ samples with a high degree of PC MRO and CRN Si samples.

Comparison of calculations from the models with available experimental data shows that PC-K1 is the most physically realistic model of the medium range order present in $\mathrm{Si}$ and Ge. Its subtle MRO is not enough to disturb its $g_{2}(r)$, which is a good match for the experimental data, but it is enough to provide the best available match for the fluctuation microscopy data. CRN-K1 has good agreement with experiment in its $g_{2}(r)$, but it does not match the fluctuation microscopy data. PC-K1 also has the closest match of the PC models to the experimental VDOS and Raman $I_{\mathrm{TA}} / I_{\mathrm{TO}}$ ratios. The larger-grain PC models, PC-K1.5 and PC-K2, have more MRO than real materials, as seen by the extra peaks in $g_{2}(r)$, and the failure to match the fluctuation microscopy and $I_{\mathrm{TA}} / I_{\mathrm{TO}}$ ratio data.

Calculations of the electronic properties show that only PC-K1 annealed with EDIP and PC-K1EDIP1 approach having a reasonable electronic band gap. This can be attributed to the high density of coordination defects present in the SW models. This demonstrates that whereas the paracrystalline medium-range order of the models is quite robust, the detailed defect structure is more sensitive to the choice of the empirical potential used and the annealing/quenching procedure employed in model preparation.

Consequently, further modeling effort is required to produce more realistic PC models of $a$-Si. On one hand, larger and more diverse models will allow us to find a better representation of the subtle MRO reflected in the fluctuation microscopy and vibrational experiments and to explore the thermodynamics of PC materials reflected in the PC grain size and density stability condition. On the other hand, a more through MD relaxation is needed to provide models with even lower defect densities. It may be possible to employ a variant of the WWW bond-switching algorithm, ${ }^{37}$ which produces perfectly fourfold coordinated structures by construction. Further simulations may also elucidate the nature of the grain boundary regions and their effect on the localization of the vibrational modes. 


\section{ACKNOWLEDGMENTS}

The authors thank Dr. John Abelson for introducing D.A.D., S.M.N., and N.Z. to the other authors, for providing the impetus for our collaboration, and for several helpful discussions. They also thank Hyun-Chul Jin for preparation of the $a$-Si sample used in the variable coherence microscopy experiments, Dr. Sjoerd Roorda for the experimental $a$-Si $g_{2}(r)$ data, and Dr. Normand Mousseau for the EDOS data of the ART model. P.M.V. acknowledges the support of the National Science Foundation under Grant No. DMR 9703906. D.A.D. and S.M.N. acknowledge the support of the NSF under Grants Nos. DMR 96-04921 and DMR 96-18789. P.K. acknowledges the support of the NSF under Grant No. DMR 00-74273.

${ }^{1}$ W. Schülke, Philos. Mag. B 43, 451 (1981).

${ }^{2}$ R. J. Temkin, W. Paul, and G. A. N. Connell, Adv. Phys. 22, 581 (1973).

${ }^{3}$ K. Laaziri, S. Kycia, S. Roorda, M. Chicoine, J. L. Robertson, J. Wang, and S. C. Moss, Phys. Rev. Lett. 82, 3460 (1999); Phys. Rev. B 60, 13520 (1999).

${ }^{4}$ A. Uhlherr and S. R. Elliot, Philos. Mag. B 71, 611 (1995); P. H. Gaskell and D. J. Wallis, Phys. Rev. Lett. 76, 66 (1996).

${ }^{5}$ S. R. Elliot, Adv. Phys. 38, 1 (1989).

${ }^{6}$ M. Benfatto, A. Bianconi, I. Davoli, J. Garcia, A. Marcelli, C. R. Natoli, and S. Stizza, J. Non-Cryst. Solids 77-78, 1325 (1985); A. Di Cicco, A. Bianconi, C. Coluzza, and P. Rudolf, ibid. 116, 27 (1990).

${ }^{7}$ W. A. Kamitakahara, H. R. Shanks, F. McClelland, U. Buchenau, F. Gompf, and L. Pintschovius, Phys. Rev. Lett. 52, 644 (1984).

${ }^{8}$ W. A. Kamitakahara, C. M. Soukoulis, H. R. Snaks, U. Buchenau, and G. S. Grest, Phys. Rev. B 36, 6539 (1987).

${ }^{9}$ D. Beeman, R. Tsu, and M. F. Thorpe, Phys. Rev. B 32, 874 (1985).

${ }^{10}$ A. P. Sokolov and A. P. Shebanin, Sov. Phys. Semicond. 24, 720 (1990).

${ }^{11}$ W. H. Zachariasen, J. Am. Chem. Soc. 51, 3841 (1932).

${ }^{12}$ P. A. Fedders, D. A. Drabold, and S. Nakhmanson, Phys. Rev. B 58, 15624 (1998); E. Kim and Y. H. Lee, ibid. 49, 1743 (1994).

${ }^{13}$ J. M. Gibson, M. M. J. Treacy, and P. J. Keblinski, J. Non-Cryst. Solids 231, 99 (1998).

${ }^{14}$ M. M. J. Treacy and J. M. Gibson, Acta Crystallogr., Sect. A: Found. Crystallogr. 52, 212 (1996).

${ }^{15}$ J. M. Gibson, M. M. J. Treacy, and P. M. Voyles, Ultramicroscopy 83, 169 (2000).

${ }^{16}$ P. M. Voyles, J. M. Gibson, and M. M. J. Treacy, J. Electron Microsc. 49, 259 (2000).

${ }^{17}$ J. M. Gibson and M. M. J. Treacy, Phys. Rev. Lett. 78, 1074 (1997).

${ }^{18}$ J. M. Gibson, M. M. J. Treacy, P. M. Voyles, H-C. Jin, and J. R. Abelson, Appl. Phys. Lett. 73, 3093 (1998).

${ }^{19}$ M. M. J. Treacy, P. M. Voyles, and J. M. Gibson, J. Non-Cryst. Solids 266, 150 (2000).

${ }^{20}$ M. L. Rudee and A. Howie, Philos. Mag. 25, 1001 (1972); A. Howie, O. L. Krivanek, and M. L. Rudee, ibid. 27, 235 (1973).

${ }^{21}$ A. Howie, J. Non-Cryst. Solids 31, 41 (1978).

${ }^{22}$ P. Keblinski, S. R. Phillpot, D. Wolf, and H. Gleiter, Acta Mater. 45, 987 (1997).
${ }^{23}$ F. H. Stillinger and T. A. Weber, Phys. Rev. B 31, 5262 (1985).

${ }^{24}$ W. D. Luedtke and U. Landman, Phys. Rev. B 40, 1164 (1989).

${ }^{25}$ M. Z. Bazant, E. Kaxiras, and J. F. Justo, Phys. Rev. B 56, 8542 (1997).

${ }^{26}$ P. Keblinski, R. K. Dash, M. Bazant, and M. M. J. Treacy (unpublished).

${ }^{27}$ J. G. Kirkwood, J. Chem. Phys. 7, 506 (1939).

${ }^{28}$ G. T. Barkema and N. Mousseau, Phys. Rev. Lett. 77, 4358 (1996); F. Finkemeier and W. von Niessen, Phys. Rev. B 58, 4473 (1998).

${ }^{29}$ G. Morell, R. S. Katiyar, S. Z. Weisz, J. Jia, J. Shinar, and I. Balberg, J. Appl. Phys. 78, 5120 (1995).

${ }^{30}$ Electron microscopists define $k=1 / \lambda$ for historic reasons, so the $k$ scale here is divided by $1 / 2 \pi$ from the conventional $\mathrm{x}$-ray diffraction units.

${ }^{31}$ What we call a ring here is called a fundamental circuit by O'Keeffe (Ref. 33). This definition tends to weight towards larger ring sizes in comparison with other definitions of a ring.

${ }^{32}$ This same basic concept has been previously developed using different nomenclature and methodology by Hobbs et al. (Ref. 35).

${ }^{33}$ M. O. O'Keeffe and B. G. Hyde, Crystal Structures: 1. Patterns and Symmetry (Mineralogical Society of America Monograph, Washington DC, 1996)

${ }^{34}$ M. M. J. Treacy, K. H. Randall, S. Rao, J. A. Perry, and D. J. Chadi, Z. Kristallogr. 212, 768 (1997).

${ }^{35}$ C. S. Marians and L. W. Hobbs, J. Non-Cryst. Solids 124, 242 (1990); L. W. Hobbs, C. E. Jesurum, V. Pulim, and B. Berger, Philos. Mag. A 78, 679 (1998).

${ }^{36}$ M. Marinov and N. Zotov, Phys. Rev. B 55, 2938 (1997).

${ }^{37}$ F. Wooten, K. Winer, and D. Weaire, Phys. Rev. Lett. 54, 1392 (1985).

${ }^{38}$ J. Fortner and J. S. Lannin, Phys. Rev. B 39, 5527 (1989).

${ }^{39}$ R. Alben, D. Weaire, J. E. Smith, Jr., and M. H. Brodsky, Phys. Rev. B 11, 2271 (1975)

${ }^{40}$ R. J. Bell and D. C. Hibbins-Butler, J. Phys. C 9, 2955 (1976).

${ }^{41}$ K. Winer, Phys. Rev. B 35, 2366 (1987).

${ }^{42}$ R. Biswas, A. M. Bouchard, W. A. Kamitakahara, G. S. Grest, and C. M. Soukoulis, Phys. Rev. Lett. 60, 2280 (1988).

${ }^{43}$ K. Ishibashi, K. Tsumuraya, and S. Nakata, J. Chem. Phys. 101, 1412 (1994).

${ }^{44}$ V. A. Luchnikov, N. N. Medvedev, A. Appelhagen, and A. Geiger, Mol. Phys. 88, 1337 (1996).

${ }^{45}$ J. L. Feldman, P. B. Allen, and S. R. Bickham, Phys. Rev. B 59, 3551 (1999).

${ }^{46}$ J. Fabian and P. B. Allen, Phys. Rev. Lett. 79, 1885 (1997).

${ }^{47}$ G. T. Barkema and N. Moussseau, Phys. Rev. Lett. 77, 4358 (1996); Phys. Rev. E 57, 2419 (1998); Phys. Rev. B 62, 4985 (2000).

${ }^{48}$ N. Zotov, M. Marinov, N. Mousseau, and G. Barkema, J. Phys.: Condens. Matter 11, 9647 (1999).

${ }^{49}$ S. T. Kshirsagar and J. S. Lannin, Phys. Rev. B 25, 2916 (1982).

${ }^{50}$ J. S. Lannin, L. J. Pilione, S. Kshirsagar, R. Messier, and R. C. Ross, Phys. Rev. B 26, 3506 (1982).

${ }^{51}$ S. M. Nakhmanson and D. A. Drabold, J. Non-Cryst. Solids 266-269, 156 (2000)

${ }^{52}$ S. M. Nakhmanson and D. A. Drabold, Phys. Rev. B 61, 5376 (2000).

${ }^{53}$ O. F. Sankey and D. J. Niklewski, Phys. Rev. B 40, 3979 (1989); O. F. Sankey and D. A. Drabold, Bull. Am. Phys. Soc. 36, 924 (1991).

${ }^{54}$ N. Bernstein, M. J. Mehl, D. A. Papaconstantopoulos, N. I. Papanicolauo, M. Z. Bazant, and E. Kaxiras, Phys. Rev. B 62, 4477 (2000). 